\title{
Strong first order electroweak phase transition in the CP-conserving 2HDM revisited
}

\author{
P. Basler, ${ }^{a}$ M. Krause, ${ }^{a}$ M. Mühlleitner, ${ }^{a}$ J. Wittbrodt ${ }^{a, b}$ and A. Wlotzka ${ }^{a}$ \\ ${ }^{a}$ Institute for Theoretical Physics, Karlsruhe Institute of Technology, \\ Wolfgang-Gaede-Str. 1, 76131 Karlsruhe, Germany \\ ${ }^{b}$ Deutsches Elektronen-Synchrotron DESY, \\ Notkestraße 85, D-22607 Hamburg, Germany \\ E-mail: philipp.basler@kit.edu, marcel.krause@kit.edu, \\ milada.muehlleitner@kit.edu, jonas.wittbrodt@desy.de, \\ alexander.wlotzka@kit.edu
}

ABSTRACT: The discovery of the Higgs boson by the LHC experiments ATLAS and CMS has marked a milestone for particle physics. Yet, there are still many open questions that cannot be answered within the Standard Model (SM). For example, the generation of the observed matter-antimatter asymmetry in the universe through baryogenesis can only be explained qualitatively in the SM. A simple extension of the SM compatible with the current theoretical and experimental constraints is given by the 2-Higgs-Doublet Model (2HDM) where a second Higgs doublet is added to the Higgs sector. We investigate the possibility of a strong first order electroweak phase transition in the CP-conserving 2HDM type I and type II where either of the CP-even Higgs bosons is identified with the SM-like Higgs boson. The renormalisation that we apply on the loop-corrected Higgs potential allows us to efficiently scan the $2 \mathrm{HDM}$ parameter space and simultaneously take into account all relevant theoretical and up-to-date experimental constraints. The 2HDM parameter regions found to be compatible with the applied constraints and a strong electroweak phase transition are analysed systematically. Our results show that there is a strong interplay between the requirement of a strong phase transition and collider phenomenology with testable implications for searches at the LHC.

Keywords: Beyond Standard Model, Higgs Physics

ARXIV EPRINT: 1612.04086 


\section{Contents}

1 Introduction 1

2 The effective potential 3

2.1 The CP-conserving 2-Higgs-Doublet Model 3

2.2 One-loop effective potential at finite temperature 5

$\begin{array}{llr}3 & \text { Renormalisation } & 8\end{array}$

4 Numerical analysis $\quad 11$

4.1 Minimisation of the effective potential 11

$\begin{array}{ll}4.2 \text { Constraints and parameter scan } & 12\end{array}$

5 Results $\quad \mathbf{1 5}$

5.1 Type I: parameter sets with $h \equiv h_{125} \quad 16$

$\begin{array}{ll}5.2 \text { Type II: parameter sets with } h \equiv h_{125} & 19\end{array}$

5.3 Type I: parameter sets with $H \equiv h_{125} \quad 22$

5.4 Type II: parameter sets with $H \equiv h_{125}$

6 Conclusions 26

A Masses with thermal corrections $\quad \mathbf{2 8}$

$\begin{array}{lll}\text { A.1 Fermion masses } & 28\end{array}$

$\begin{array}{lll}\text { A.2 Gauge boson masses } & 29\end{array}$

A.3 Masses of the Higgs bosons 29

\section{Introduction}

In 2012 the LHC experiments ATLAS and CMS announced the discovery of the long-sought Higgs boson [1-4]. Although it looks very SM-like [5-8] it is quite possible that it is the scalar particle of a Higgs sector beyond the SM (BSM). Despite the success of the SM, which has been tested to highest precision at previous and current colliders, there are still a lot of open questions that cannot be answered within the SM and call for new physics (NP) extensions. One of the unanswered problems is the origin of the observed matterantimatter asymmetry of the universe [9]. Electroweak (EW) baryogenesis is an elegant mechanism to explain this asymmetry [10-18], which is related to physics at the weak scale, establishing a link between collider phenomenology and cosmology. The asymmetry can be generated provided the EW phase transition (PT) taking place in the early universe is of strong first order $[16,18]$ and that all three Sakharov conditions [19] are fulfilled, namely baryon number violation, $\mathrm{C}$ and $\mathrm{CP}$ violation and departure from the thermal equilibrium. 
The strong first order PT, proceeding through bubble formation, suppresses the baryon number violating sphaleron transitions in the false vacuum [20,21]. CP-violating reflections of top quarks from the bubble wall produce a hypercharge asymmetry which is converted into a baryon asymmetry in the false vacuum. This asymmetry is transferred to the true vacuum when it passes the bubble wall [22], provided there is departure from the thermal equilibrium. Although in the SM all three Sakharov conditions are fulfilled, the electroweak PT is not of first order [23-26]. Not only the Higgs boson mass is too large [27, 28], but in addition the $\mathrm{CP}$ violation of the SM from the Cabibbo-Kobayashi-Maskawa matrix is too small $[18,22,29]$. This calls for physics BSM. Among the plethora of NP extensions the $2 \mathrm{HDM}[30,31]$ belongs to the simplest models that are in accordance with present experimental constraints. Its Higgs sector features five physical Higgs bosons, three neutral and two charged ones. Their contributions to the effective Higgs potential can strengthen the PT and in addition introduce new sources of $\mathrm{CP}$ violation. Previous studies have shown that 2HDMs provide a good framework for successful baryogenesis [32-47] (see [48-52] for studies in the CP-violating 2HDM).

In this work we will investigate the implications of a strong first order PT required by baryogenesis on the LHC Higgs phenomenology in the framework of the CP-conserving 2HDM. For this purpose we compute the one-loop corrected effective potential at finite temperature [53-55] including daisy resummations for the bosonic masses [56] in two different approximations for the treatment of the thermal masses $[57,58]$. The renormalisation of the loop-corrected potential is chosen such that not only the vacuum expectation value (VEV) and all physical Higgs boson masses, but, for the first time, also all mixing matrix elements remain at their tree-level values. This allows to efficiently scan the whole 2HDM parameter space with the tree-level masses and mixing angles as input and at the same time test the compatibility of the model with the theoretical and experimental constraints. The points passing these tests will be investigated with respect to a first order PT. The loop-corrected Higgs potential will be minimised at increasing non-zero temperature to find the vacuum expectation value $v_{c}$ at the critical temperature $T_{c}$, defined as the temperature where two degenerate global minima exist. A value of $v_{c} / T_{c},{ }^{1}$ larger than one is indicative for a strong first order PT $[13,62]$. In our analysis we will discard points leading to a 2-stage PT $[63,64]$. We will perform a systematic and comprehensive investigation of the 2HDM in four configurations, given by the 2HDM type I and type II where either the lighter or the heavier of the two CP-even Higgs bosons is identified with the SM-like Higgs boson. We will test the compatibility of the model with both the experimental constraints and a strong EW phase transition. The thus delineated regions in the parameter space will be further investigated with respect to their implications for collider phenomenology. We find that the link between cosmology and high-energy collider constraints provides a powerful tool to further constrain the allowed parameter regions of the 2HDM. At the same time, the demand for a strong first order PT leads to testable consequences at the collider experiments.

The outline of the paper is as follows: in section 2 we introduce our notation and provide the loop-corrected effective potential at non-vanishing temperature. In the subsequent

\footnotetext{
${ }^{1}$ For discussions on the gauge dependence of $v_{c} / T_{c}$, see $[54,59-61]$.
} 
section 3 we describe in detail the renormalisation procedure, which is chosen such that at zero temperature the tree-level position of the minimum and the masses and mixing matrix elements of the scalar particles are preserved by the one-loop potential. Using the Higgs boson masses and mixing angles as input parameters, this simplifies the verification of the compatibility of the model with the Higgs data. In section 4 the basic elements of our numerical analysis are described, namely the minimisation procedure of the effective potential in 4.1 and, in 4.2, the details of the scan in the $2 \mathrm{HDM}$ parameter space together with the applied theoretical and experimental constraints. Section 5 is devoted to our results. We present the parameter regions compatible with the applied constraints and a strong first order PT, and we then analyse the implications for collider phenomenology. We end in section 6 with our conclusions. The paper is accompanied by an appendix containing the formulae for the masses of the relevant particles and, where appropriate, for the daisy resummed mass corrections.

\section{The effective potential}

In this section we provide the loop-corrected effective potential of the CP-conserving 2HDM for non-vanishing temperature. First, we set our notation by introducing the model under investigation.

\subsection{The CP-conserving 2-Higgs-Doublet Model}

In terms of the two $\mathrm{SU}(2)_{L}$ Higgs doublets $\Phi_{1}$ and $\Phi_{2}$,

$$
\Phi_{1}=\left(\begin{array}{c}
\phi_{1}^{+} \\
\phi_{1}^{0}
\end{array}\right) \quad \text { and } \quad \Phi_{1}=\left(\begin{array}{c}
\phi_{2}^{+} \\
\phi_{2}^{0}
\end{array}\right),
$$

the tree-level potential of the $2 \mathrm{HDM}$ with a softly broken $\mathbb{Z}_{2}$ symmetry, under which the doublets transform as $\Phi_{1} \rightarrow \Phi_{1}, \Phi_{2} \rightarrow-\Phi_{2}$, reads

$$
\begin{aligned}
V_{\text {tree }}= & m_{11}^{2} \Phi_{1}^{\dagger} \Phi_{1}+m_{22}^{2} \Phi_{2}^{\dagger} \Phi_{2}-\left[m_{12}^{2} \Phi_{1}^{\dagger} \Phi_{2}+\text { h.c. }\right]+\frac{1}{2} \lambda_{1}\left(\Phi_{1}^{\dagger} \Phi_{1}\right)^{2}+\frac{1}{2} \lambda_{2}\left(\Phi_{2}^{\dagger} \Phi_{2}\right)^{2} \\
& +\lambda_{3}\left(\Phi_{1}^{\dagger} \Phi_{1}\right)\left(\Phi_{2}^{\dagger} \Phi_{2}\right)+\lambda_{4}\left(\Phi_{1}^{\dagger} \Phi_{2}\right)\left(\Phi_{2}^{\dagger} \Phi_{1}\right)+\left[\frac{1}{2} \lambda_{5}\left(\Phi_{1}^{\dagger} \Phi_{2}\right)^{2}+\text { h.c. }\right] .
\end{aligned}
$$

The mass parameters $m_{11}^{2}$ and $m_{22}^{2}$ and the couplings $\lambda_{1 \ldots 4}$ are real parameters of the model. The mass and coupling parameters $m_{12}^{2}$ and $\lambda_{5}$ can in general be complex, thereby offering new sources of explicit CP violation in the Higgs sector. We take them to be real as we work in the CP-conserving 2HDM. After EW symmetry breaking the two Higgs doublets acquire VEVs $\bar{\omega}_{i} \in \mathbb{R}(i=1,2,3)$, about which the Higgs fields can be expanded in terms of the charged CP-even and CP-odd fields $\rho_{i}$ and $\eta_{i}$, and the neutral CP-even and CP-odd fields $\zeta_{i}$ and $\psi_{i}, i=1,2$,

$$
\begin{aligned}
\Phi_{1} & =\frac{1}{\sqrt{2}}\left(\begin{array}{c}
\rho_{1}+\mathrm{i} \eta_{1} \\
\bar{\omega}_{1}+\zeta_{1}+\mathrm{i} \psi_{1}
\end{array}\right) \\
\Phi_{2} & =\frac{1}{\sqrt{2}}\left(\begin{array}{c}
\rho_{2}+\mathrm{i} \eta_{2} \\
\bar{\omega}_{2}+\mathrm{i} \bar{\omega}_{3}+\zeta_{2}+\mathrm{i} \psi_{2}
\end{array}\right),
\end{aligned}
$$


where, without loss of generality, the complex part of the VEVs has been rotated to the second doublet exclusively. Denoting the VEVs of our present vacuum at zero temperature by ${ }^{2}$

$$
\left.v_{i} \equiv \bar{\omega}_{i}\right|_{T=0},
$$

we set

$$
v_{3}=0,
$$

whereas the remaining two VEVs are related to the SM VEV by

$$
v_{1}^{2}+v_{2}^{2} \equiv v^{2} .
$$

Introducing the angle $\beta$ through

$$
\tan \beta=\frac{v_{2}}{v_{1}}
$$

we have

$$
v_{1}=v \cos \beta \quad \text { and } \quad v_{2}=v \sin \beta .
$$

Through the complex part of the VEV, $\bar{\omega}_{3}$, we include the possibility of generating at one-loop and/or non-zero temperature a global minimum that is CP-violating. ${ }^{3}$ The angle $\beta$ coincides with the angle of the rotation matrix

$$
R(\beta)=\left(\begin{array}{cc}
\cos \beta & \sin \beta \\
-\sin \beta & \cos \beta
\end{array}\right)
$$

from the gauge to the mass eigenstates of the charged Higgs sector, and also of the neutral $\mathrm{CP}$-odd sector. The physical states of the charged sector are given by the charged Higgs bosons $H^{ \pm}$with mass $m_{H^{ \pm}}$and the charged Goldstone bosons $G^{ \pm}$which are massless at zero temperature,

$$
\left(\begin{array}{c}
G^{ \pm} \\
H^{ \pm}
\end{array}\right)=R(\beta)\left(\begin{array}{l}
\phi_{1}^{ \pm} \\
\phi_{2}^{ \pm}
\end{array}\right) \text {. }
$$

For the neutral CP-odd fields $\psi_{1}$ and $\psi_{2}$ the same rotation yields the physical states $A$ with mass $m_{A}$ and the neutral Goldstone boson $G^{0}$, massless at zero temperature,

$$
\left(\begin{array}{c}
G^{0} \\
A
\end{array}\right)=R(\beta)\left(\begin{array}{l}
\psi_{1} \\
\psi_{2}
\end{array}\right) .
$$

Finally, in the neutral CP-even sector the rotation with the angle $\alpha$ transforms the fields $\zeta_{1}$ and $\zeta_{2}$ into the two physical CP-even Higgs bosons $H$ and $h$ with masses $m_{H}$ and $m_{h}$, respectively,

$$
\left(\begin{array}{c}
H \\
h
\end{array}\right)=R(\alpha)\left(\begin{array}{l}
\zeta_{1} \\
\zeta_{2}
\end{array}\right) .
$$

\footnotetext{
${ }^{2}$ Strictly speaking, $T=2.7 \mathrm{~K}$. Setting $T=0$ does not make a difference numerically.

${ }^{3}$ In the $2 \mathrm{HDM}$ we can have three different types of minima: the normal EW breaking one, a CP-breaking minimum, and a charge-breaking (CB) vacuum. It has been shown that, at tree level, minima which break different symmetries cannot coexist [65-67]. This means that, if a normal minimum exists, all CP or CB stationary points are proven to be saddle points. Recent studies of the Inert 2HDM at one-loop level [68], which apply the effective potential approach, indicate that these statements may not be true any more once higher order corrections are included. We therefore allow for the possibility of a CP-breaking vacuum. Including the possibility of a charge breaking Higgs VEV makes the present analysis considerably more complex.
} 


\begin{tabular}{|lcccc|}
\hline & Type I & Type II & Lepton-Specific & Flipped \\
\hline Up-type quarks & $\Phi_{2}$ & $\Phi_{2}$ & $\Phi_{2}$ & $\Phi_{2}$ \\
Down-type quarks & $\Phi_{2}$ & $\Phi_{1}$ & $\Phi_{2}$ & $\Phi_{1}$ \\
Leptons & $\Phi_{2}$ & $\Phi_{1}$ & $\Phi_{1}$ & $\Phi_{2}$ \\
\hline
\end{tabular}

Table 1. Classification of the Yukawa sector in the 2HDM according to the couplings of the fermions to the Higgs doublets.

In the minimum of the potential eq. (2.2) the following minimum conditions have to be fulfilled,

$$
\left.\frac{\partial V_{\text {tree }}}{\partial \Phi_{a}^{\dagger}}\right|_{\Phi_{i}=\left\langle\Phi_{i}\right\rangle} \stackrel{!}{=} 0 \quad a, i \in\{1,2\}
$$

with the brackets denoting the Higgs field values in the minimum, i.e. $\left\langle\Phi_{i}\right\rangle=\left(0, v_{i} / \sqrt{2}\right)$ at $T=0$. This results in two equations

$$
\begin{aligned}
& m_{11}^{2}=m_{12}^{2} \frac{v_{2}}{v_{1}}-\frac{v_{1}^{2}}{2} \lambda_{1}-\frac{v_{2}^{2}}{2}\left(\lambda_{3}+\lambda_{4}+\lambda_{5}\right) \\
& m_{22}^{2}=m_{12}^{2} \frac{v_{1}}{v_{2}}-\frac{v_{2}^{2}}{2} \lambda_{2}-\frac{v_{1}^{2}}{2}\left(\lambda_{3}+\lambda_{4}+\lambda_{5}\right) .
\end{aligned}
$$

Exploiting the minimum conditions of the potential at zero temperature, we use the following set of independent parameters of the model,

$$
m_{h}, m_{H}, m_{A}, m_{H^{ \pm}}, m_{12}^{2}, \alpha, \tan \beta, v .
$$

Due to the imposed $\mathbb{Z}_{2}$ symmetry each of the up-type quarks, down-type quarks and charged leptons can only couple to one of the Higgs doublets so that flavour-changing neutral currents at tree level are avoided. The possible combinations of Yukawa couplings of the Higgs bosons to up-type quarks, down-type quarks or charged leptons are classified as type I, type II, lepton-specific and flipped and are defined in table 1 . The resulting couplings of the fermions normalised to the SM couplings can be found in [69]. In this work we focus on real $2 \mathrm{HDM}$ of type I and type II.

\subsection{One-loop effective potential at finite temperature}

The one-loop contributions $V_{1}$ to the effective potential consist of two parts: the ColemanWeinberg (CW) contribution $V_{\mathrm{CW}}$ [53] which is already present at zero temperature, and the contribution $V_{T}$ accounting for the thermal corrections at finite temperature $T$. The one-loop corrected effective potential then reads

$$
V=V_{\text {tree }}+V_{1} \equiv V_{\text {tree }}+V_{\mathrm{CW}}+V_{T} .
$$

The tree-level potential is given in eq. (2.2) with the doublet $\Phi_{1}$ replaced by the classical constant field configuration $\Phi_{1}^{c}=\left(0, \omega_{1} / \sqrt{2}\right)$ and $\Phi_{2}$ by $\Phi_{2}^{c}=\left(0,\left(\omega_{2}+i \omega_{3}\right) / \sqrt{2}\right)$. The Coleman-Weinberg potential in the $\overline{\mathrm{MS}}$ scheme is given by [55]

$$
V_{\mathrm{CW}}(\{\omega\})=\sum_{i} \frac{n_{i}}{64 \pi^{2}}(-1)^{2 s_{i}} m_{i}^{4}(\{\omega\})\left[\log \left(\frac{m_{i}^{2}(\{\omega\})}{\mu^{2}}\right)-c_{i}\right],
$$


where the sum extends over the Higgs and Goldstone bosons, the massive gauge bosons, the longitudinal photon and the fermions, $i=h, H, A, H^{ \pm}, G^{0}, G^{ \pm}, W^{ \pm}, Z, \gamma_{L}, f$ $(f=e, \mu, \tau, u, c, t, d, s, b){ }^{4}$ The $m_{i}^{2}$ is the respective eigenvalue for the particle $i$ of the mass matrix squared expressed through the tree-level relations in terms of $\omega_{i}(i=1,2,3)$. The explicit formulae can be found in appendix A. The sum also includes the Goldstone bosons. Although we work in the Landau gauge, where they are massless at $T=0$, they can acquire a mass if the mass eigenvalues are determined at field configurations other than the tree-level VEVs at $T=0$, which is required in the minimisation procedure. Moreover, due to temperature corrections specified below, the masses of the Goldstones and the longitudinal photon can be non-zero, which enforces also the inclusion of $\gamma_{L}$ in the sum. Note, that due to the choice of the Landau gauge there are no ghost contributions. The variable $s_{i}$ denotes the spin of the particle, $n_{i}$ represents the number of degrees of freedom. Also for later use, we define the degrees of freedom of all particles involved in the model. These are the neutral scalars $\Phi^{0} \equiv h, H, A, G^{0}$, the charged scalars $\Phi^{ \pm} \equiv H^{ \pm}, G^{ \pm}$, the leptons $l$, the quarks $q$ and the longitudinal and transversal gauge bosons, $V_{L} \equiv Z_{L}, W_{L}, \gamma_{L}$ and $V_{T} \equiv Z_{T}, W_{T}, \gamma_{T}$, with the respective $n_{i}$,

$$
\begin{aligned}
n_{\Phi^{0}} & =1, \quad n_{\Phi^{ \pm}}=2, \quad n_{l}=4, \quad n_{q}=12, \\
n_{W_{T}} & =4, \quad n_{W_{L}}=2, \quad n_{Z_{T}}=2, \quad n_{Z_{L}}=1, \\
n_{\gamma_{T}} & =2, \quad n_{\gamma_{L}}=1 .
\end{aligned}
$$

In the $\overline{\mathrm{MS}}$ scheme employed here, the constants $c_{i}$ read

$$
c_{i}= \begin{cases}\frac{5}{6}, & i=W^{ \pm}, Z, \gamma \\ \frac{3}{2}, & \text { otherwise } .\end{cases}
$$

We fix the renormalisation scale $\mu$ by $\mu=v=246.22 \mathrm{GeV}$.

In the thermal corrections $V_{T}$ we include the daisy resummation [56] of the $n=0$ Matsubara modes of the longitudinal components of the gauge bosons $W_{L}, Z_{L}, \gamma_{L}$ and the bosons $\Phi^{0}, \Phi^{ \pm}$, which adds to their masses at non-zero temperature the Debye corrections given in appendix A. The thermal contributions $V_{T}$ to the potential can be written as $[54,55]$

$$
V^{T}=\sum_{k} n_{k} \frac{T^{4}}{2 \pi^{2}} J_{ \pm}^{(k)} .
$$

The sum extends over $k=W_{L}, Z_{L}, \gamma_{L}, W_{T}, Z_{T}, \Phi^{0}, \Phi^{ \pm}, f$. Note, that the Goldstone bosons and the longitudinal part of the photon, which are massless at $T=0$, acquire a mass at finite temperature and are included in the sum. Denoting the mass eigenvalue including the thermal corrections for the particle $k$ by $\bar{m}_{k}, J_{ \pm}^{(k)}$ is given by (see e.g. [70])

$$
J_{ \pm}^{(k)}= \begin{cases}J_{-}\left(\frac{m_{k}^{2}}{T^{2}}\right)-\frac{\pi}{6}\left(\frac{\bar{m}_{k}^{3}}{T^{3}}-\frac{m_{k}^{3}}{T^{3}}\right) k & =W_{L}, Z_{L}, \gamma_{L}, \Phi^{0}, \Phi^{ \pm} \\ J_{-}\left(\frac{m_{k}^{2}}{T^{2}}\right) & k=W_{T}, Z_{T} \\ J_{+}\left(\frac{m_{k}^{2}}{T^{2}}\right) & k=f\end{cases}
$$

\footnotetext{
${ }^{4}$ Note, that we assume the neutrinos to be massless.
} 
with the thermal integrals

$$
J_{ \pm}\left(\frac{m_{k}^{2}}{T^{2}}\right)=\mp \int_{0}^{\infty} \mathrm{d} x x^{2} \log \left[1 \pm e^{-\sqrt{x^{2}+m_{k}^{2} / T^{2}}}\right],
$$

where $J_{+}\left(J_{-}\right)$applies for $k$ being a fermion (boson). For each temperature $T$ we determine the VEVs $\bar{\omega}_{i}$, i.e. the field configurations $\{\bar{\omega}\} \equiv\left\{\bar{\omega}_{1}, \bar{\omega}_{2}, \bar{\omega}_{3}\right\}$, that minimise the loop-corrected potential $V$, eq. (2.17). These enter the tree-level mass matrices such that the masses $m_{i}$ depend implicitly on the temperature $T$ through $\bar{\omega}_{i}=\bar{\omega}_{i}(T)$. The $\bar{m}_{k}$ furthermore depend explicitly on $T$ through the thermal corrections. The definition of $J_{ \pm}^{(k)}$ eq. (2.22) is the approach chosen in [57]. A different prescription for implementing the thermal corrections is proposed by [58] where the Debye corrections are included for all the bosonic thermal loop contributions, ${ }^{5}$ so that we have

$$
J_{ \pm}^{(k)}=\left\{\begin{array}{l}
J_{-}\left(\frac{\bar{m}_{k}^{2}}{T^{2}}\right) k=W_{L}, Z_{L}, \gamma_{L}, \Phi^{0}, \Phi^{ \pm} \\
J_{-}\left(\frac{m_{k}^{2}}{T^{2}}\right) k=W_{T}, Z_{T} \\
J_{+}\left(\frac{m_{k}^{2}}{T^{2}}\right) k=f .
\end{array}\right.
$$

In this case, the Debye corrected masses are also used in the CW potential eq. (2.18) [50]. We refer to the first approach, eq. (2.22), as 'Arnold-Espinosa' and to the second one, i.e. eq. (2.24) together with $V_{\mathrm{CW}}$ including the thermal corrections in the bosonic masses, as 'Parwani' method. The two approaches differ in the organisation of the perturbative series and hence by higher order terms. The 'Arnold-Espinosa' method consistently implements the thermal masses at one-loop level in the high-temperature expansion, leading to eq. (2.22). The 'Parwani' method admixes higher-order contributions, which at one-loop level could lead to dangerous artefacts. Therefore, in the discussion of our results we will apply the 'Arnold-Espinosa' method. The 'Parwani' method will be used only to make contact to previous results in the literature.

Since in the minimisation procedure the numerical evaluation of the integral eq. (2.23) at each configuration in $\{\omega\}$ and $T$ is very time consuming, the integrals $J_{ \pm}$are approximated by a series in $x^{2} \equiv m^{2} / T^{2}$. For small $x^{2}$ we use [48]

$$
\begin{aligned}
J_{+, \mathrm{s}}\left(x^{2}, n\right)= & -\frac{7 \pi^{4}}{360}+\frac{\pi^{2}}{24} x^{2}+\frac{1}{32} x^{4}\left(\log x^{2}-c_{+}\right) \\
& -\pi^{2} x^{2} \sum_{l=2}^{n}\left(-\frac{1}{4 \pi^{2}} x^{2}\right)^{l} \frac{(2 l-3) ! ! \zeta(2 l-1)}{(2 l) ! !(l+1)}\left(2^{2 l-1}-1\right) \\
J_{-, \mathrm{s}}\left(x^{2}, n\right)= & -\frac{\pi^{4}}{45}+\frac{\pi^{2}}{12} x^{2}-\frac{\pi}{6}\left(x^{2}\right)^{3 / 2}-\frac{1}{32} x^{4}\left(\log x^{2}-c_{-}\right) \\
& +\pi^{2} x^{2} \sum_{l=2}^{n}\left(-\frac{1}{4 \pi^{2}} x^{2}\right)^{l} \frac{(2 l-3) ! ! \zeta(2 l-1)}{(2 l) ! !(l+1)}
\end{aligned}
$$

with

$$
c_{+}=3 / 2+2 \log \pi-2 \gamma_{E} \quad \text { and } \quad c_{-}=c_{+}+2 \log 4,
$$

\footnotetext{
${ }^{5}$ For a discussion and comparison, see also [48, 50].
} 
where $\gamma_{E}$ denotes the Euler-Mascheroni constant, $\zeta(x)$ the Riemann $\zeta$-function and $(x)$ !! the double factorial. For large $x^{2}$ the expansion for both fermions and bosons reads [48]

$$
J_{ \pm, 1}\left(x^{2}, n\right)=-\exp \left(-\left(x^{2}\right)^{1 / 2}\right)\left(\frac{\pi}{2}\left(x^{2}\right)^{3 / 2}\right)^{1 / 2} \sum_{l=0}^{n} \frac{1}{2^{l} l !} \frac{\Gamma(5 / 2+l)}{\Gamma(5 / 2-l)}\left(x^{2}\right)^{-l / 2}
$$

with $\Gamma(x)$ denoting the Euler Gamma function. In order to interpolate between the two approximations, first the point is determined where the derivatives of the low- and hightemperature expansions can be connected continuously. We then add a small finite shift to the small $x^{2}$ expansion such that also the two expansions themselves are connected continuously. We denote the values of $x^{2}$ where the connection is performed by $x_{+}^{2}$ and $x_{-}^{2}$ and the corresponding shifts by $\delta_{ \pm}$for the fermionic and bosonic contributions, respectively. They are given by

$$
\begin{array}{ll}
x_{+}^{2}=2.2161, & \delta_{+}=-0.015603, \\
x_{-}^{2}=9.4692, & \delta_{-}=0.0063109 .
\end{array}
$$

We find that for small $x^{2}$ the expansion $J_{+, \mathrm{s}}$ for fermions approximates the exact result well by including terms of up to order $n=4$, while for bosons this is the case for $n=3$ in $J_{-, \mathrm{s}}$. For large $x^{2}$, the integral is well approximated by $n=3$ in both the fermion and the boson case, $J_{ \pm, 1}$. This way, the deviation of the approximate results from the numerical evaluation of the integrals is less than two percent. The above approximations eqs. (2.25)-(2.28) are only valid for $m^{2} \geq 0$. For bosons this is not necessarily the case as the eigenvalues of the mass matrix of the neutral Higgs bosons can become negative depending on the configuration $\{\omega\}$ and the temperature in the minimisation procedure. If this happens, the value of the integral $J_{-}$, given by eq. (2.23), is set to the real part of its numerical evaluation which is the relevant contribution when extracting the global minimum [71]. ${ }^{6}$ In practice, we evaluated the integral numerically at several equidistant points in $m^{2} / T^{2}<0$, and in the minimisation procedure we use the result obtained from the linear interpolation between these points, which leads to a significant speed-up. We explicitly verified that the difference between the exact and the interpolated result is negligible for a sufficiently large range of $m^{2} / T^{2}$.

\section{Renormalisation}

The Coleman-Weinberg potential, eq. (2.18), in the one-loop effective potential eq. (2.17) contributes already at $T=0$, so that the masses and mixing angles obtained from the oneloop effective potential differ from those extracted from the tree-level potential eq. (2.2). The loop-corrected masses obtained in this way correspond to the full one-loop corrected masses in the approximation of vanishing external momenta. When we test for compatibility of the model with the experimental constraints the loop-corrected masses and the loop-corrected mixing angles, which enter the couplings, have to be taken into account. For an efficient scan over the parameter space of the model in terms of the input parameters

\footnotetext{
${ }^{6}$ Note, that negative masses squared correspond to a negative curvature of the potential, implying a local maximum and not a minimum.
} 
eq. (2.16), however, it is more convenient to have the one-loop masses and angles directly as inputs, i.e. they should be the same as the tree-level ones. This can be achieved by an appropriate renormalisation prescription, which will be described in the following.

The Coleman-Weinberg potential given in eq. (2.18) has already been renormalised in the $\overline{\mathrm{MS}}$ scheme. We modify this scheme by including finite terms in the counterterm potential that ensure the one-loop corrected masses and, for the first time, also the mixing matrix elements to be equal to the tree-level ones. ${ }^{7}$ Introducing counterterms for each of the parameters of the tree-level potential eq. (2.2), the counterterm potential $V_{\mathrm{CT}}$ added to the one-loop effective potential eq. (2.17),

$$
\tilde{V}=V+V_{\mathrm{CT}}=V_{\text {tree }}+V_{\mathrm{CW}}+V_{T}+V_{\mathrm{CT}},
$$

reads

$$
\begin{aligned}
V_{\mathrm{CT}}= & \delta m_{11}^{2} \frac{\omega_{1}^{2}}{2}+\delta m_{22}^{2} \frac{\omega_{2}^{2}+\omega_{3}^{2}}{2}-\delta m_{12}^{2} \omega_{1} \omega_{2}+\frac{\delta \lambda_{1}}{8} \omega_{1}^{4}+\frac{\delta \lambda_{2}}{8}\left(\omega_{2}^{2}+\omega_{3}^{2}\right)^{2} \\
& +\left(\delta \lambda_{3}+\delta \lambda_{4}\right) \frac{\omega_{1}^{2}\left(\omega_{2}^{2}+\omega_{3}^{2}\right)}{4}+\delta \lambda_{5} \frac{\omega_{1}^{2}\left(\omega_{2}^{2}-\omega_{3}^{2}\right)}{4}
\end{aligned}
$$

The complete potential of eq. (3.1) will be minimised to find the global minimum at a given temperature $T$. As stated above, the counterterms $\delta p$ for the parameters $p$ of the tree-level potential contain only the finite pieces, as the divergent ones have already been absorbed by the $\overline{\mathrm{MS}}$ renormalised $V_{\mathrm{CW}}$. We renormalise the effective potential such that at $T=0$ the tree-level position of the minimum yields a local minimum, which is checked to be the global one numerically. Furthermore, through our renormalisation the masses and mixing angles of the scalar particles are preserved at their tree-level values by the one-loop potential. The corresponding renormalisation conditions are imposed at $T=0$, which is where we test for the compatibility with the experimental constraints. The position of the minimum is determined by the first derivative of the potential, whereas the masses and angles result from the second derivative, namely the mass matrix. Formulae for both the first and the second derivatives of the CW potential in the Landau gauge have been derived in [72]. We employ these formulae in the gauge basis to calculate the required derivatives. Consequently, for the renormalisation we also express the counterterm potential and the tree-level potential in the gauge basis. The renormalisation conditions for the first derivatives are then given by $(i=1, \ldots, 8)$

$$
\left.\partial_{\phi_{i}} V_{\mathrm{CT}}(\phi)\right|_{\phi=\left\langle\phi^{c}\right\rangle_{T=0}}=-\left.\partial_{\phi_{i}} V_{\mathrm{CW}}(\phi)\right|_{\phi=\left\langle\phi^{c}\right\rangle_{T=0}}
$$

with

$$
\phi_{i} \equiv\left\{\rho_{1}, \eta_{1}, \rho_{2}, \eta_{2}, \zeta_{1}, \psi_{1}, \zeta_{2}, \psi_{2}\right\},
$$

\footnotetext{
${ }^{7}$ Previous works included only the VEVs and (subsets of) the masses in the renormalisation conditions and required them to be equal to their tree-level values [46-50]. In models with extended Higgs sectors, the mixing angles, which enter all Higgs boson observables through the Higgs couplings, are crucial for the interpretation of the results. They are determined from the diagonalisation of the loop-corrected mass matrix. The renormalisation of the mixing matrix elements to their tree-level values guarantees that the relevant quantities and observables constraining the model can be tested with the tree-level input parameters.
} 
and $\left\langle\phi^{c}\right\rangle_{T=0}$ denoting the field configuration in the minimum at $T=0$,

$$
\left\langle\phi^{c}\right\rangle_{T=0}=\left(0,0,0,0, v_{1}, 0, v_{2}, 0\right) .
$$

This results in two non-trivial conditions for the tree-level minimum at $T=0$ to be a CPconserving extremum also at the one-loop level. In order to ensure that both the masses and the mixing angles remain at their tree-level values the complete $8 \times 8$ mass matrix of the scalar sector should be preserved at its tree-level value by the renormalised one-loop potential. This is achieved by demanding $(i, j=1, \ldots, 8)$

$$
\left.\partial_{\phi_{i}} \partial_{\phi_{j}} V_{\mathrm{CT}}(\phi)\right|_{\phi=\left\langle\phi^{c}\right\rangle_{T=0}}=-\left.\partial_{\phi_{i}} \partial_{\phi_{j}} V_{\mathrm{CW}}(\phi)\right|_{\phi=\left\langle\phi^{c}\right\rangle_{T=0}} .
$$

However, since we have only eight counterterms and after imposing eq. (3.3) we are left with six to be set, the resulting system of equations is overconstrained and cannot in general be solved. This means that we cannot renormalise all masses and mixing angles to exactly match their tree-level values. We therefore pursue the following approach: both the tree-level and the one-loop mass matrix are rotated to the mass basis with the tree-level rotation matrix. From the resulting $8 \times 8$ matrix we extract only the $2 \times 2$ submatrix, that corresponds to the physical charged Higgs bosons, and the $3 \times 3$ submatrix for the neutral Higgs bosons. In the CP-conserving case treated here, the latter decomposes into a $2 \times 2$ matrix for the CP-even Higgs bosons $h$ and $H$, and the entry for the pseudoscalar $A$. On these submatrices the renormalisation conditions are imposed, so that we have

$$
\left.\left.\partial_{\phi_{i}} \partial_{\phi_{j}} V_{\mathrm{CT}}(\phi)\right|_{\phi=\left\langle\phi^{c}\right\rangle_{T=0}}\right|_{\text {mass }} ^{H^{ \pm}}=-\left.\left.\partial_{\phi_{i}} \partial_{\phi_{j}} V_{\mathrm{CW}}(\phi)\right|_{\phi=\left\langle\phi^{c}\right\rangle_{T=0}}\right|_{\text {mass }} ^{H^{ \pm}}
$$

and

$$
\left.\left.\partial_{\phi_{i}} \partial_{\phi_{j}} V_{\mathrm{CT}}(\phi)\right|_{\phi=\left\langle\phi^{c}\right\rangle_{T=0}}\right|_{\text {mass }} ^{h, H, A}=-\left.\left.\partial_{\phi_{i}} \partial_{\phi_{j}} V_{\mathrm{CW}}(\phi)\right|_{\phi=\left\langle\phi^{c}\right\rangle_{T=0}}\right|_{\text {mass }} ^{h, H, A} .
$$

The subscript 'mass' indicates that the mass matrix in the gauge basis is rotated into the mass eigenbasis by means of the rotation matrix that diagonalises the tree-level mass matrix. The superscripts $H^{ \pm}$and $h, H, A$ indicate that from the resulting matrix only the $2 \times 2$ block for the physical charged Higgs bosons and the $3 \times 3$ block for the physical neutral Higgs bosons is considered, respectively. Equations (3.7) and (3.8) provide five independent non-trivial renormalisation conditions. ${ }^{8}$ Together with the two renormalisation conditions from eq. (3.3) we have altogether seven renormalisation conditions to fix eight renormalisation constants, cf. eq. (3.2), so that one renormalisation constant is left for determination. Inspecting the counterterm potential eq. (3.2), we observe that the counterterms $\delta \lambda_{3}$ and $\delta \lambda_{4}$ only appear as sum. Hence, we choose to use only one of them and set $\delta \lambda_{4}=0$. The remaining seven renormalisation constants are fixed by the conditions eqs. (3.3), (3.7) and (3.8).

We find that these renormalisation conditions allow us to preserve the minimum, the masses and the mixing angles of the Higgs sector at their tree-level values up to a very

\footnotetext{
${ }^{8}$ After application of eq. (3.3) some matrix elements of the extracted submatrices are linear combinations of other matrix elements so that we do not have further conditions.
} 
good approximation. Taking into account numerical uncertainties, the minimum at oneloop remains at $v \pm 2 \mathrm{GeV}$, and all masses and mixing angles are preserved up to tiny numerical fluctuations.

Equations (3.7) and (3.8) require the second derivative of the $\mathrm{CW}$ potential. It is a well-known problem that this derivative leads to infrared divergences for the Goldstone bosons in the Landau gauge [46, 48, 50, 72-74]. In order to circumvent this problem, in [48] the logarithm is redefined to capture on-shell effects regularising the divergence while in $[46,47,50]$ a non-vanishing infrared mass for the Goldstones is employed to regulate the divergence. In the effective potential approach itself, which is the approximation of the full theory at vanishing external momenta, it is not possible to cancel these divergences. Building up the complete self-energy of the Higgs bosons from the effective potential and the momentum-dependent parts obtained by a diagrammatic calculation, however, it becomes apparent that the infrared divergences from the Goldstone contributions cancel between the CW part and the momentum-dependent part [72, 74, 75]. Taking the limit of vanishing external momenta afterwards we arrive at a finite expression for the second derivative of the $\mathrm{CW}$ potential. This cancellation was checked explicitly using the results from the diagrammatic calculation performed in [76, 77]. In practice, this result can be obtained directly from the effective potential approach by regularising the logarithmic divergence with a regulator mass and then discarding the terms proportional to this logarithm [72]. The obtained results are independent of the regulator mass and reflect the correct contributions present in the effective potential approach. ${ }^{9}$

\section{Numerical analysis}

\subsection{Minimisation of the effective potential}

The electroweak $\mathrm{PT}$ is considered to be strong if the ratio between the VEV $v_{c}$ at the critical temperature $T_{c}$ and the critical temperature $T_{c}$ is larger than one $[13,62]$,

$$
\xi_{c} \equiv \frac{v_{c}}{T_{c}} \geq 1
$$

The value $v$ at a given temperature $T$ is obtained as

$$
v(T)=\sqrt{\bar{\omega}_{1}^{2}+\bar{\omega}_{2}^{2}+\bar{\omega}_{3}^{2}} .
$$

Remind that $\bar{\omega}_{i}$ are the field configurations that minimise the loop-corrected effective potential at non-zero temperature. The critical temperature $T_{c}$ is defined as the temperature where the potential has two degenerate minima. For the determination of $T_{c}$ the effective potential together with the counterterm potential, eq. (3.1), is minimised numerically at a given temperature $T$. In a first order electroweak PT the VEV jumps from $v=v_{c}$ at the temperature $T_{c}$ to $v=0$ for $T>T_{c}$. In order to double-check the results of the minimisation procedure, we apply two different minimisation algorithms. One is the active

\footnotetext{
${ }^{9}$ Note, that this problem does not occur for the higher-order contributions to the Higgs masses resulting from loops with a photon inside, as the only class of diagrams possibly leading to infrared divergences (diagrams with a scalar and a vector boson in the loop) vanishes for zero external momenta.
} 
CMA-ES algorithm as implemented in libcmaes [78]. This algorithm finds the global minimum of a given function. As termination criterion we choose the relative tolerance of the value of the effective potential between two iterations to be smaller than $10^{-5}$. The other algorithm that has been used is the local Nelder-Mead-Simplex algorithm from the GNU Scientific Library [79] (gsl_multimin_fminimizer_nmsimplex2), also with a tolerance of $10^{-5}$. For a given temperature, we start with 500 randomly distributed points in the interval $\omega_{1,2,3} \in[-500,500] \mathrm{GeV}$ for which we compute the minimum of the potential. Note that we have included $\bar{\omega}_{3}$ in eq. (2.4) for the sake of generality. The candidates for the global minimum obtained with the two algorithms are compared to each other and the one with the lower value of the effective potential is chosen as the global minimum. Although there may be local minima that are CP-violating we find that in the global minimum $\bar{\omega}_{3}$ always vanishes up to numerical fluctuations at both $T=0$ and $T=T_{c}$. Hence we will not comment on it any further. In order to determine the critical temperature $T_{c}$ where the phase transition takes place, we employ a bisection method in the temperature $T$, starting with the determination of the minimum at the temperatures $T_{S}=0 \mathrm{GeV}$ and ending at $T_{E}=300 \mathrm{GeV}$. The minimisation procedure is terminated when the interval containing $T_{c}$ is smaller than $10^{-2} \mathrm{GeV}$. The temperature $T_{c}$ is then set to the lower bound of the final interval. We exclude parameter points that do not satisfy $|v(T=0)-246.22 \mathrm{GeV}| \leq 2 \mathrm{GeV}$, and parameter points where no PT is found for $T \leq 300 \mathrm{GeV} \cdot{ }^{10}$ Moreover, we only retain parameter points with $T_{c}>10 \mathrm{GeV}$.

The complete calculation and implementation was checked against an independent calculation in Mathematica. Profiting from significant speed-up, the implementation above was used for the results presented in this work.

\subsection{Constraints and parameter scan}

We determine the value of $\xi_{c}$ only for those points that are compatible with theoretical and experimental constraints. In order to obtain viable data sets we use ScannerS [80,81] to perform extensive scans in the $2 \mathrm{HDM}$ parameter space and check for compatibility with the constraints. The program verifies if the tree-level potential is bounded from below by applying the conditions given in [82] and checks for tree-level perturbative unitarity as described in [83]. In the $\mathrm{CP}$-conserving $2 \mathrm{HDM}$ investigated here, the requirement that the neutral CP-even tree-level minimum is the global one is tested through a simple condition [84]. The consistency with the EW precision constraints has been checked through the oblique parameters $S, T$ and $U$ [85] by applying the general procedure for extended Higgs sectors as described in $[86,87]$ and demanding for compatibility with the SM fit [88] within $2 \sigma$, including correlations. Constraints applied to the charged sector of the $2 \mathrm{HDM}$ are based on results from the measurement of $R_{b}$ [89, 90] and $B \rightarrow X_{s} \gamma$ [90-92] including the recent calculation [93] that enforces

$$
m_{H \pm}>480 \mathrm{GeV}
$$

\footnotetext{
${ }^{10}$ For temperatures $T_{c} \geq 246 \mathrm{GeV}$ the $\mathrm{VEV}$ would have to be larger than $246 \mathrm{GeV}$ in order to fulfill the criterion of a strong first order PT. By choosing $300 \mathrm{GeV}$ we apply an additional safety margin.
} 
in type II models. In type I models the bound is much weaker and more strongly dependent on $\tan \beta$. Note, that the results from LEP [94] and the LHC $[95,96]^{11}$ require the charged Higgs mass to be above $\mathcal{O}(100 \mathrm{GeV})$ depending on the model type. For the check of the compatibility with the Higgs data we need the Higgs production cross sections normalised to the corresponding SM values and the Higgs branching ratios. The latter have been computed with HDECAY version 6.51 [98-100]. This program includes the state-of-the-art higher order QCD corrections and off-shell decays. The Higgs production cross sections through gluon fusion and $b$-quark fusion at the LHC have been obtained at NNLO QCD from an interface with SusHi $[100,101]$ and normalised to the corresponding SM value at NNLO QCD. The cross section ratio for associated production with a heavy quark pair has been taken at LO. In the ratio involving CP-even Higgs bosons the QCD corrections drop out. This is not the case for the pseudoscalar. For associated production with top quarks the cross section is very small. The associated production with bottom quarks can be important for large values of $\tan \beta$. However, here the QCD corrections in the associated production of the pseudoscalar with the bottom quark pair almost cancel against those of the SM counterpart due to the nearly realised chiral limit for the small $b$-quark masses. The remaining processes through gauge boson fusion and Higgs radiation off a $W^{ \pm}$or $Z$ boson only apply for a CP-even Higgs boson so that here the QCD corrections drop out when normalised to the SM cross section. Since not all EW corrections have been provided for the $2 \mathrm{HDM}$ so far they are consistently neglected in all production and decay processes. Agreement with the exclusion bounds from LHC Higgs searches has been tested with HiggsBounds [102-104]. Compatibility with the observed signal of the $125 \mathrm{GeV}$ Higgs boson has been verified by calculating the reduced signal strengths and checking against the two times one sigma bounds in the six parameter fit of [105]. Further details on the various checks can be found in [81]. ${ }^{12}$

For the minimisation procedure we only use parameter points that are in agreement with the described theoretical and experimental constraints. In order to find viable parameter points we perform a scan in the $2 \mathrm{HDM}$ parameter space given by the input parameters eq. (2.16). The SM VEV given by the Fermi constant $G_{F}$ through $v=1 / \sqrt{\sqrt{2} G_{F}}$, has been fixed to

$$
v=246.22 \mathrm{GeV} .
$$

The mixing angle $\alpha$ is varied in the theoretically allowed region, i.e.

$$
-\frac{\pi}{2} \leq \alpha<\frac{\pi}{2}
$$

In all scans, one of the masses of the CP-even Higgs bosons has been fixed to [106]

$$
m_{h_{125}}=125.09 \mathrm{GeV} \text {. }
$$

This is the Higgs boson we identify with the SM-like Higgs boson discovered at the LHC, and we denote it by $h_{125}$. We performed two separate scans for the cases where the

\footnotetext{
${ }^{11}$ The recent ATLAS results [97] have not been translated into bounds so far.

${ }^{12}$ The respective experimental values cited there have been replaced by the latest experimental results.
} 


\begin{tabular}{|c|c|c|c|c|c|c|}
\hline \multirow[t]{2}{*}{ \# points } & \multirow[t]{2}{*}{$m_{h}$} & $m_{H}$ & $m_{A}$ & $m_{H^{ \pm}}$ & $m_{12}^{2}$ & $\tan (\beta)$ \\
\hline & & \multicolumn{2}{|c|}{ in $\mathrm{GeV}$} & \multicolumn{3}{|c|}{ in $\mathrm{GeV}^{2}$} \\
\hline 1000000 & $m_{h_{125}}$ & $130-1000$ & $30-1000$ & $65-1000$ & $0-5 \times 10^{5}$ & $1-35$ \\
\hline 100000 & $30-120$ & $m_{h_{125}}$ & $30-1000$ & $65-1000$ & $0-5 \times 10^{5}$ & $1-35$ \\
\hline
\end{tabular}

Table 2. Parameter ranges for the scan performed in the 2HDM type I. The first column specifies the number of points generated.

\begin{tabular}{|rcccccc|}
\hline \# points & $m_{h}$ & $m_{H}$ & $\begin{array}{c}m_{A} \\
\text { in GeV }\end{array}$ & $m_{H^{ \pm}}$ & $\begin{array}{c}m_{12}^{2} \\
\text { in } \mathrm{GeV}^{2}\end{array}$ & $\tan (\beta)$ \\
\hline 1000000 & $m_{h_{125}}$ & $130-1000$ & $30-1000$ & $480-1000$ & $0-5 \times 10^{5}$ & $0.1-35$ \\
100000 & $30-120$ & $m_{h_{125}}$ & $450-1000$ & $480-1000$ & $0-5 \times 10^{5}$ & $0.1-35$ \\
\hline
\end{tabular}

Table 3. Parameter ranges for the scan in the 2HDM type II. The first column specifies the number of points generated.

lighter or the heavier of the two CP-even Higgs bosons is identified with the SM-like Higgs, i.e. $m_{h}=m_{h_{125}}$ and $m_{H}=m_{h_{125}}$, respectively. The scan ranges for the remaining parameters are given in table 2 in case of type I and in table 3 for type II. In our scans we required the neighboring non-SM-like Higgs masses to deviate by at least $5 \mathrm{GeV}$ from $125.09 \mathrm{GeV}$, in order to avoid degenerate Higgs signals. The input masses for the non-SMlike neutral Higgs bosons were chosen within $30 \mathrm{GeV}$ and $1000 \mathrm{GeV}$ and the input mass for the charged Higgs boson within $65 \mathrm{GeV}$ and $1000 \mathrm{GeV}$ to cover most of the parameter space which is potentially interesting for phenomenology and accessible by experiments. The parameter $m_{12}^{2}$ is constrained by the tree-level global minimum condition to be positive. The upper limits on $\tan \beta$ and $m_{12}^{2}$ have been set by choice, but as we observe later, most of the points compatible with the constraints and a strong PT are found for rather small $\tan \beta$ so that the chosen upper limit does not pose a strong constraint. Type-specific choices for the ranges are the lower bound on $\tan \beta$ in type $\mathrm{I}$ and the lower bound on $m_{H^{ \pm}}$in type II. They have been chosen such that they already leave out part of the parameter space that is excluded by the constraints from $B \rightarrow X_{s} \gamma$ measurements. Moreover, in type II the lower bound on $m_{A}$ in the second set, where $H \equiv h_{125}$, is motivated by the fact that fulfilling constraints on the oblique parameters requires one Higgs to be in vicinity of the charged Higgs boson. In the second set this can only be the pseudoscalar Higgs $A$.

For the SM parameters we have chosen the following values: apart from the computation of the oblique parameters, where we use the fine structure constant at zero momentum transfer,

$$
\alpha_{\mathrm{EM}}^{-1}(0)=137.0359997
$$

the fine structure constant is taken at the $Z$ boson mass scale [107],

$$
\alpha_{\mathrm{EM}}^{-1}\left(M_{Z}^{2}\right)=128.962 .
$$


The massive gauge boson masses are chosen as [107, 108]

$$
M_{W}=80.385 \mathrm{GeV} \quad \text { and } \quad M_{Z}=91.1876 \mathrm{GeV},
$$

the lepton masses as $[107,108]$

$$
m_{e}=0.510998928 \mathrm{MeV}, \quad m_{\mu}=105.6583715 \mathrm{MeV}, \quad m_{\tau}=1.77682 \mathrm{GeV},
$$

and the light quark masses are set following [109] to

$$
m_{u}=100 \mathrm{MeV}, \quad m_{d}=100 \mathrm{MeV}, \quad m_{s}=100 \mathrm{MeV} .
$$

For consistency with the ATLAS and CMS analyses the on-shell top quark mass

$$
m_{t}=172.5 \mathrm{GeV}
$$

has been taken as recommended by the LHC Higgs Cross section Working Group (HXSWG) [108, 110]. The charm and bottom quark on-shell masses are [108]

$$
m_{c}=1.51 \mathrm{GeV} \quad \text { and } \quad m_{b}=4.92 \mathrm{GeV} .
$$

We take the CKM matrix to be real, with the CKM matrix elements given by $[107]^{13}$

$$
V_{\mathrm{CKM}}=\left(\begin{array}{ccc}
V_{u d} & V_{u s} & V_{u b} \\
V_{c d} & V_{c s} & V_{c b} \\
V_{t d} & V_{t s} & V_{t b}
\end{array}\right)=\left(\begin{array}{ccc}
0.97427 & 0.22536 & 0.00355 \\
-0.22522 & 0.97343 & 0.0414 \\
0.00886 & -0.0405 & 0.99914
\end{array}\right) .
$$

\section{Results}

We now turn to the presentation of our results. We will discuss the specific features of the $2 \mathrm{HDM}$ parameter space that is compatible with the theoretical and experimental constraints and at the same time provides a strong first order PT. We will show results both for the type I and the type II 2HDM. For comparison with results in the literature, we show one plot where we have applied the 'Parwani' method in the treatment of the thermal masses, cf. subsection 2.2. In the remaining discussion, however, we apply the 'Arnold-Esinosa' method for reasons discussed in [57] and alluded to in section 2.2. We will discuss scenarios where the lighter of the CP-even Higgs bosons is identified with the discovered Higgs boson, i.e. $h \equiv h_{125}$, and where $H \equiv h_{125}$.

For the interpretation of our results some general considerations on first order PTs are in order. The value of $\xi_{c}$ is proportional to the couplings of the light bosonic particles to the SM-like Higgs boson, and it decreases with the Higgs boson mass [70]. The additional Higgs bosons in the $2 \mathrm{HDM}$ spectrum allow for large trilinear bosonic couplings, in contrast to the SM, where bosonic couplings are only due to the weak gauge couplings between the Higgs boson and the EW gauge bosons. In the 2HDM, the second CP-even Higgs

\footnotetext{
${ }^{13}$ In the computation of the counterterms we choose $V_{\mathrm{CKM}}=\mathbb{1}$ for simplicity. The impact of this choice on the counterterms and thereby on the potential and its minimisation is negligible.
} 

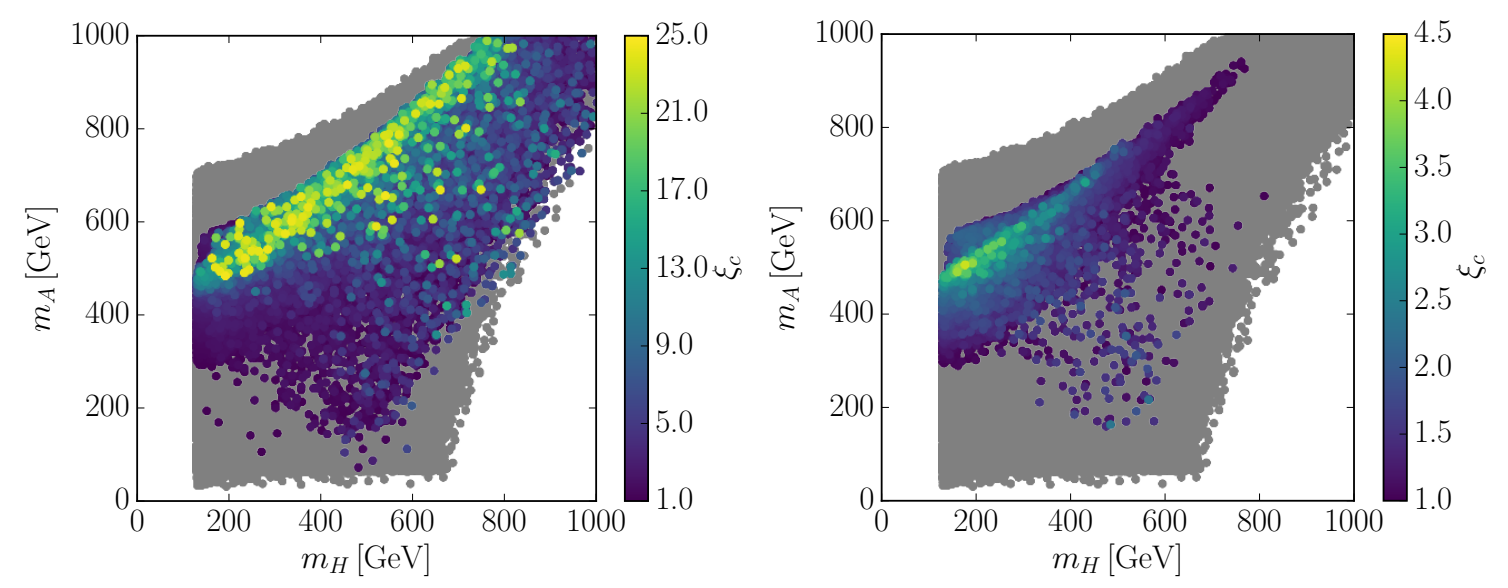

Figure 1. Type I, $h \equiv h_{125}$ : results in the $m_{A}$ versus $m_{H}$-plane, showing in grey the parameter points passing all applied constraints. Points highlighted in color have a PT of strong first order, where the value of $\xi_{c}$ is indicated by the color code. Left: 'Parwani' method, right: 'ArnoldEspinosa' method.

boson with a non-vanishing VEV contributes to the PT and can reduce its strength if $H$ is not light enough. A strong electroweak PT therefore requires $H$ either to be light or to have a vanishing VEV. The latter corresponds to the alignment limit where only one of the physical Higgs bosons has a VEV [111]. Previous investigations suggest that a firstorder PT prefers a scalar spectrum, which is not too heavy $[46,47,50]$, or else a large mass splitting between the heavy scalars $[47,51]$. In the type II $2 \mathrm{HDM}$ the requirement of a light Higgs spectrum puts some tension on the model, as compatibility with the EW precision tests requires one of the non-SM-like neutral Higgs bosons to be close to $m_{H^{ \pm}}$. Charged Higgs masses below $480 \mathrm{GeV}$ on the other hand are already excluded by $B \rightarrow X_{s} \gamma$.

\subsection{Type I: parameter sets with $h \equiv h_{125}$}

We start with the analysis of the results in the 2 HDM type I. Figure 1 shows in the $m_{A}$ versus $m_{H}$ plane all parameter points that pass the applied constraints, for scenarios where $h \equiv h_{125}$. The coloured points are those for which we obtain a strong first order PT, i.e. where $\xi_{c} \geq 1$. In the treatment of the thermal masses we have applied the 'Parwani' method (left plot) in order to compare to the results of [47], where the 'Parwani' method was applied. In the right plot we show the results for the 'Arnold-Espinosa' method, which we will use in the remainder of the discussion. As can be inferred from the plots, in the 2HDM type I first order PTs are still possible taking into account the up-to-date LHC Higgs data and all theoretical constraints on the 2HDM Higgs potential. The comparison of the left and right plot, however, also shows that the results obtained for $\xi_{c}$ are significantly different when the two different approximations in the treatment of the thermal masses are applied. Overall, the regions in the parameter space compatible with $\xi_{c} \geq 1$ are smaller when the 'Arnold-Espinosa' method is applied. Furthermore, the maximum values of $\xi_{c}$ that can be obtained with the 'Parwani' method are by a factor five larger than those obtained with the 'Arnold-Espinosa' method. Working with a one-loop effective potential 

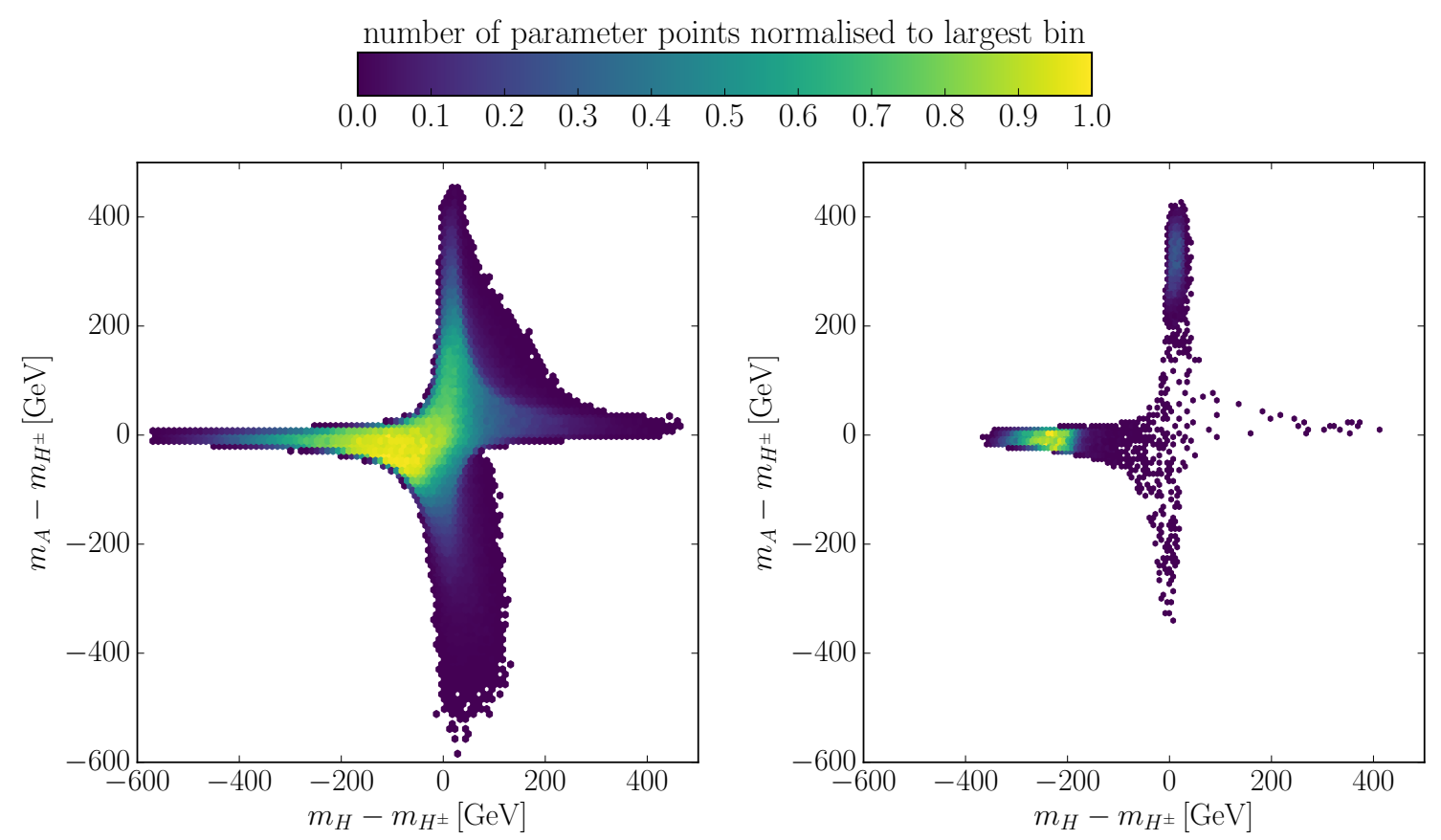

Figure 2. Type I, $h \equiv h_{125}$ : the mass difference $m_{A}-m_{H^{ \pm}}$versus $m_{H}-m_{H^{ \pm}}$. The colour code shows the relative frequency of left: all points passing the constraints; right: all points with additionally $\xi_{c} \geq 1$ ('Arnold-Espinosa' method).

only, the 'Parwani' method cannot be applied consistently, which is reflected in the very different results for both methods. Note also that the unrealistically large values for $\xi_{c}$ obtained in the 'Parwani' method imply very low critical temperatures $T_{c}$ where the phase transition takes place. This again questions the way the thermal masses are implemented so that the results of the 'Parwani' method have to be taken with care. In the following, we will only show plots for the 'Arnold-Espinosa' method.

In order to examine how the requirement of a strong first order phase transition translates into LHC Higgs phenomenology we show in figure 2 the mass differences between the non-SM-like Higgs bosons. The left plot shows the frequency of the points that pass the constraints. The right plot displays the frequency of the points when additionally a strong EW phase transition is required. As can be inferred from the left plot, the EW precision tests, namely the measurement of the $\rho$ parameter, force the mass differences between the charged Higgs boson and at least one of the non-SM-like Higgs bosons to be small and strongly favour mass spectra where all of the non- $h_{125}$ masses are close to each other. The requirement of a strong EW phase transition, however, favours scenarios where the pseudoscalar mass is close to $m_{H^{ \pm}}$with a larger mass gap relative to a lighter $H$. In figure 3 we display the relative frequencies in the $m_{A}$ versus $m_{H}$ plane for all point passing the constraints (left) and for those points which additionally fulfill $\xi_{c} \geq 1$ (right). The comparison of the two plots shows that the requirement of a strong PT favours a mass spectrum where the heaviest Higgs bosons $A$ and $H^{ \pm}$have masses around $400-500 \mathrm{GeV}$ and $m_{H} \approx 200 \mathrm{GeV}$. $H$, which acquires a VEV, should be light, so that the strength of 

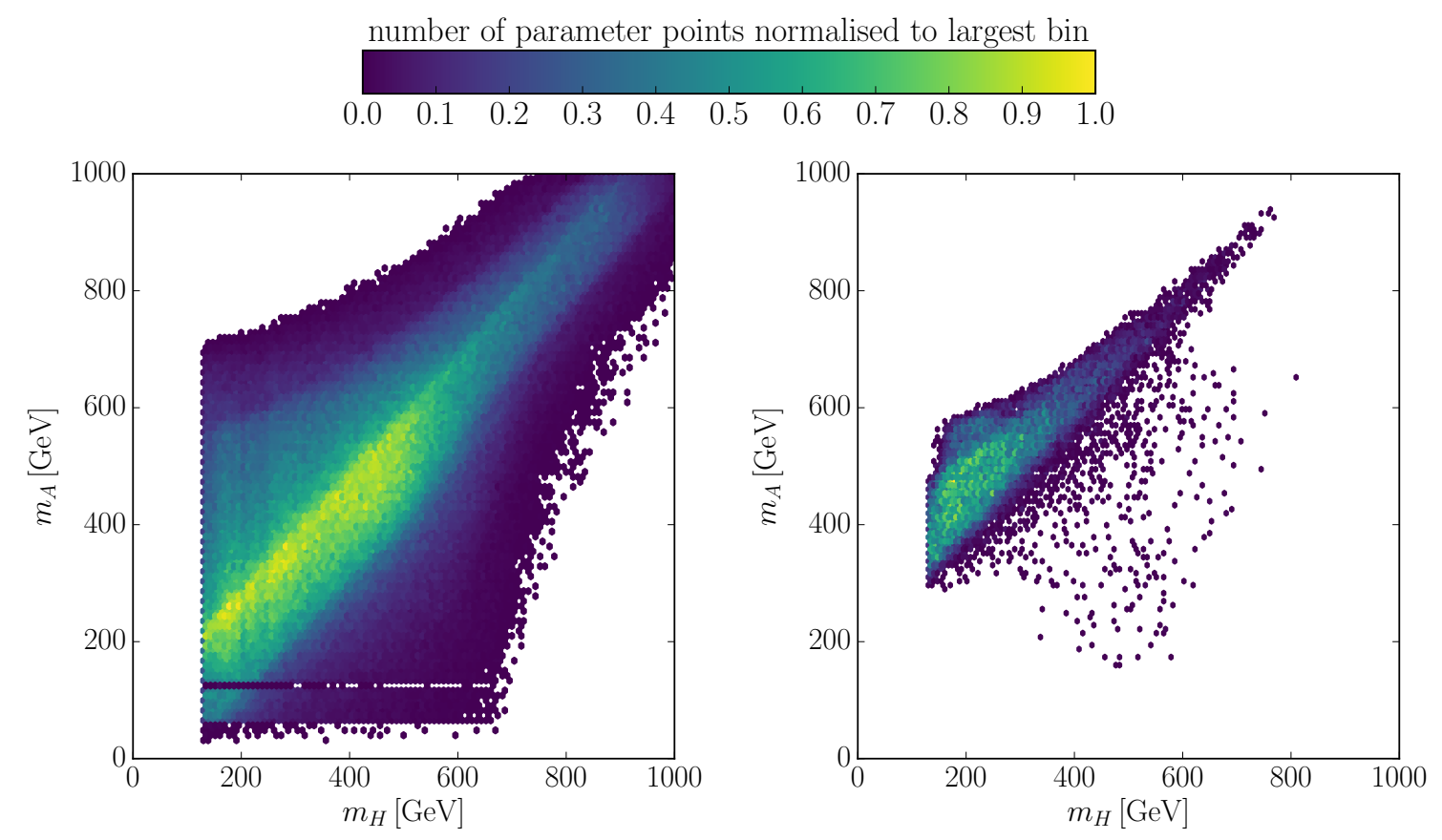

Figure 3. Type I, $h \equiv h_{125}$ : the masses $m_{A}$ versus $m_{H}$. The colour code shows the relative frequency of left: all points passing the constraints; right: all points with additionally $\xi_{c} \geq 1$ ('Arnold-Espinosa' method).

the PT is not reduced by a heavy $H$. Consequently $m_{12}^{2}$ is small, ${ }^{14}$ which means that the strength of the phase transition is governed by the quartic couplings $\lambda_{4}$ and $\lambda_{5}$, cf. also [46]. The next important mass configuration is given by scenarios where again the mass gap between $A$ and $H$ is large, but now overall pushed to higher mass values, i.e. $m_{H} \approx m_{H^{ \pm}}$ and $m_{A}-m_{H} \approx 350 \mathrm{GeV}$, cf. figure 2 (right). Since $h \equiv h_{125}$ and hence $\sin (\beta-\alpha) \approx 1$, the coupling $g_{Z A H} \sim \sin (\beta-\alpha)$ between $A, Z$ and $H$ is significant. The requirement of a strong PT prefers scenarios where the decay $A \rightarrow Z H$ is kinematically allowed so that this decay can become important. These scenarios can be searched for at the LHC, as has been found earlier in [47] and proposed by the authors as possible benchmark scenarios. Still, figure 2 demonstrates that also scenarios are compatible with $\xi_{c} \geq 1$ where all three nonSM-like Higgs bosons are close in mass or where the decay $H \rightarrow A Z$ is possible, i.e. where $m_{H}>m_{A}$ and either $m_{H}-m_{H^{ \pm}} \approx 0$ or $m_{A}-m_{H^{ \pm}} \approx 0$. While our results confirm earlier results in the literature [47,51], our results also show that a decay $A \rightarrow Z H$ is not unique for a 2HDM type I featuring a strong first order PT.

The majority of the scenarios we find is very close to the alignment limit, i.e. $\sin (\beta-\alpha) \approx 1$ with $\tan \beta$ close to its smallest possible value of about 2.5. While this is a feature resulting already from the constraints applied, the requirement of a strong PT overall pushes the Higgs rates towards SM values, as can be inferred from figure 4. It shows in grey the distribution of the Higgs signal strengths for the scenarios passing the

\footnotetext{
${ }^{14}$ The masses of the heavy Higgs bosons $\Phi=H, A, H^{ \pm}$are given by $m_{\phi}^{2}=m_{12}^{2} /(\sin \beta \cos \beta) c_{\phi}^{2}+f\left(\lambda_{i}\right) v^{2}$, where $f\left(\lambda_{i}\right)$ is a linear combination of $\lambda_{1}-\lambda_{5}$ and $c_{\phi}=1$ for $\phi=A, H^{ \pm}$and $\sin (\beta-\alpha)$ for $\phi=H$ [77].
} 

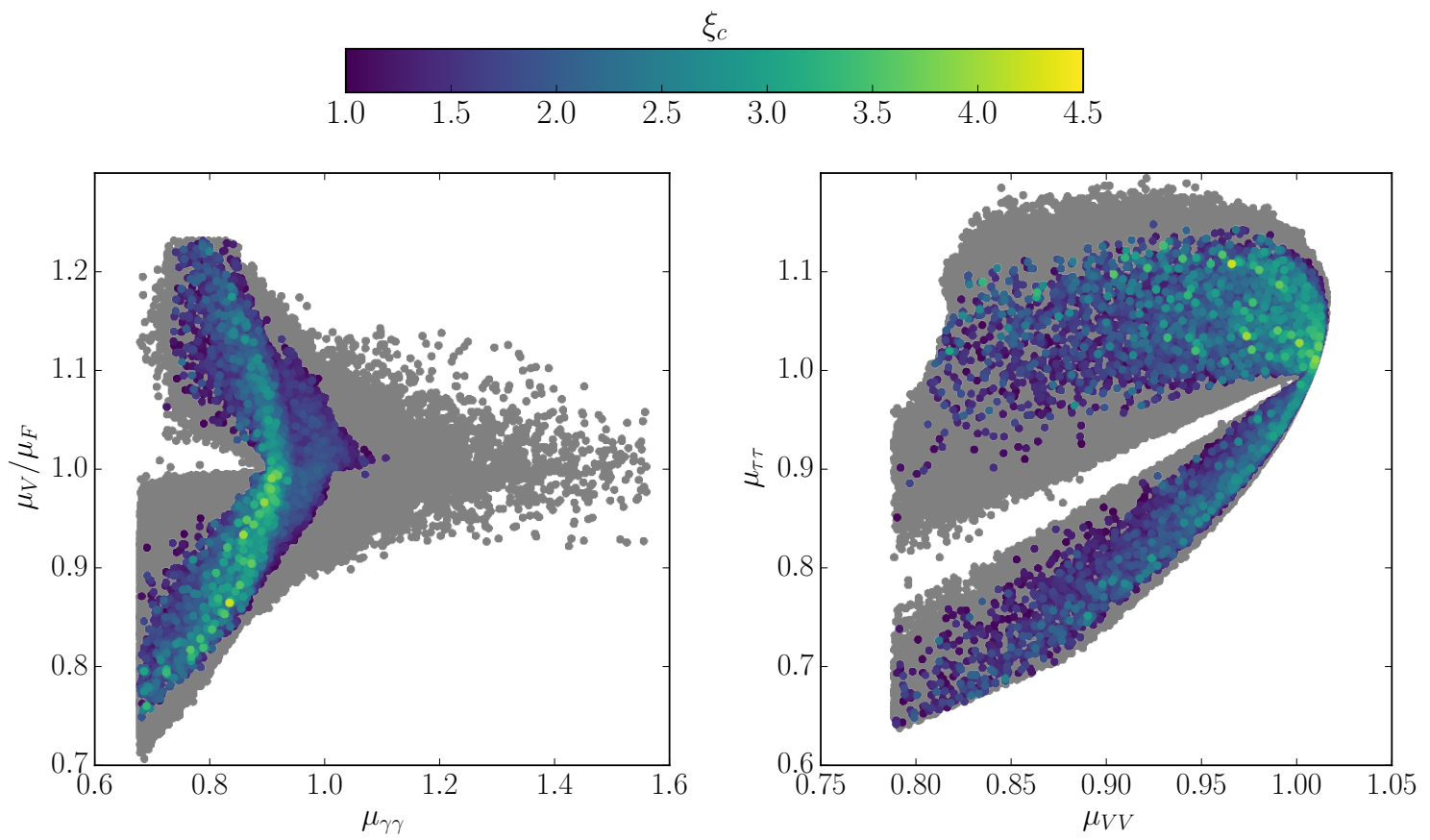

Figure 4. Type I, $h \equiv h_{125}: \mu_{V} / \mu_{F}$ versus $\mu_{\gamma \gamma}$ (left) and $\mu_{\tau \tau}$ versus $\mu_{V V}$ (right); grey: all points passing the applied constraints, colour: all points with additionally $\xi_{c} \geq 1$ ('Arnold-Espinosa' method). The colour code indicates the value of $\xi_{c}$.

constraints and in colour the scenarios that are additionally compatible with a strong PT. The colour code indicates the strength of the PT. The left plot shows $\mu_{V} / \mu_{F}$ versus $\mu_{\gamma \gamma}$ and the right one $\mu_{\tau \tau}$ versus $\mu_{V V}$. Here $\mu_{F}$ denotes the fermion initiated cross section (gluon fusion and associated production with a heavy quark pair) of the SM-like Higgs boson $\left(h_{125}\right)$ normalised to the SM, and $\mu_{V}$ the normalised production cross section through massive gauge bosons (gauge boson fusion and associated production with a vector boson). The value $\mu_{x x}$ is defined as

$$
\mu_{x x}=\mu_{F} \frac{\mathrm{BR}_{2 \mathrm{HDM}}\left(h_{125} \rightarrow x x\right)}{\mathrm{BR}_{\mathrm{SM}}\left(H_{\mathrm{SM}} \rightarrow x x\right)},
$$

where $H_{\mathrm{SM}}$ is the SM Higgs boson with mass $125 \mathrm{GeV}$. The left plot shows that for $\mu_{V} / \mu_{F}$ close to 1 , enhanced signal rates in the photonic final states with $\mu_{\gamma \gamma}$ of up to about 1.5 are still allowed. However, including the requirement for a strong first order PT the possible range of an enhanced $\mu_{\gamma \gamma}$ is strongly restricted down to $\mu_{\gamma \gamma} \approx 1.1$. On the other hand, the limits on the $\tau$ or gauge boson final states are not as significantly changed, as can be inferred from figure 4 (right).

\subsection{Type II: parameter sets with $h \equiv h_{125}$}

We now turn to the discussion of the compatibility of the 2HDM type II with the requirement of $\xi_{c} \geq 1$ for scenarios with $h=h_{125}$. Figure 5, which displays the values of $\xi_{c}$ for all parameter points compatible with our constraints, shows that also in the 2HDM type II there are scenarios allowing for a strong first order PT. The constraints from $B$-physics 


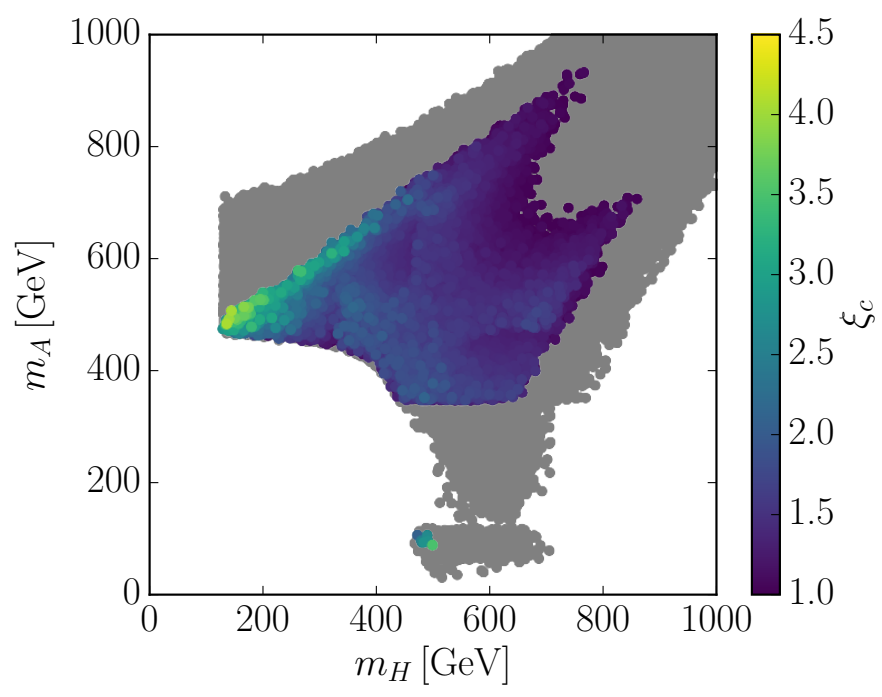

Figure 5. Type II, $h \equiv h_{125}$ : results in the $m_{A}$ versus $m_{H}$-plane, showing in grey the parameter points passing all applied constraints. Points highlighted in color have a PT of strong first order, where the value of $\xi_{c}$ is indicated by the color code ('Arnold-Espinosa' method).

observables and the EW precision tests raise the mass scale for $m_{H^{ \pm}}$and at least one of the non-SM-like Higgs bosons to higher values. For $m_{A} \lesssim 350 \mathrm{GeV}$ we only find few scenarios compatible with the experimental constraints. The pseudoscalar with $m_{A} \lesssim 350 \mathrm{GeV}$ has a significant branching ratio into $Z h$ (up to 10\%). This final state has been searched for by the LHC experiments. The resulting exclusion limits severely constrain this parameter region so that there the amount of points compatible with the experimental constraints is substantially smaller than above the top quark pair threshold where $A$ dominantly decays into $t \bar{t} .{ }^{15}$ When additionally a strong first order $\mathrm{PT}$ is required, the mass region $130 \mathrm{GeV} \lesssim m_{A} \lesssim 340 \mathrm{GeV}$ is completely excluded. As can be inferred from the plot, for these values of $m_{A}$ the heavy Higgs mass ranges between $\sim 450$ and $700 \mathrm{GeV}$. In this range the occurrence of a strong first order PT is strongly limited by deviations from the exact alignment limit at the per mille level. The small portions of the VEV assigned to $H$ by these tiny deviations already suppress the strength of the PT strongly due to the large $m_{H}$. Once $m_{H}>650 \mathrm{GeV}$, even for the parameter points extremely close to the alignment limit, the $H$ mass is finally too heavy to allow for a strong PT. The restrictions for $m_{A} \lesssim 120 \mathrm{GeV}$ on the other hand are less severe, as there are less experimental studies in this mass region so that we have more points allowed by the experimental constraints. This increases the chances of finding a strong first order PT and explains why we have some coloured points for $m_{A} \lesssim 120 \mathrm{GeV}$.

The implications of the requirement of a strong first order phase transition in the type II $2 \mathrm{HDM}$ for LHC phenomenology can be read off figure 6. Scenarios with all non-SMlike Higgs masses being close to each other are favoured by the experimental constraints,

\footnotetext{
${ }^{15}$ In type I, where also $H$ or $H^{ \pm}$can be light and hence $A \rightarrow Z H$ or $A \rightarrow W^{ \pm} H^{\mp}$ decays are possible, the LHC searches, which focus on the $A \rightarrow Z h_{125}$ decays, are less restrictive.
} 

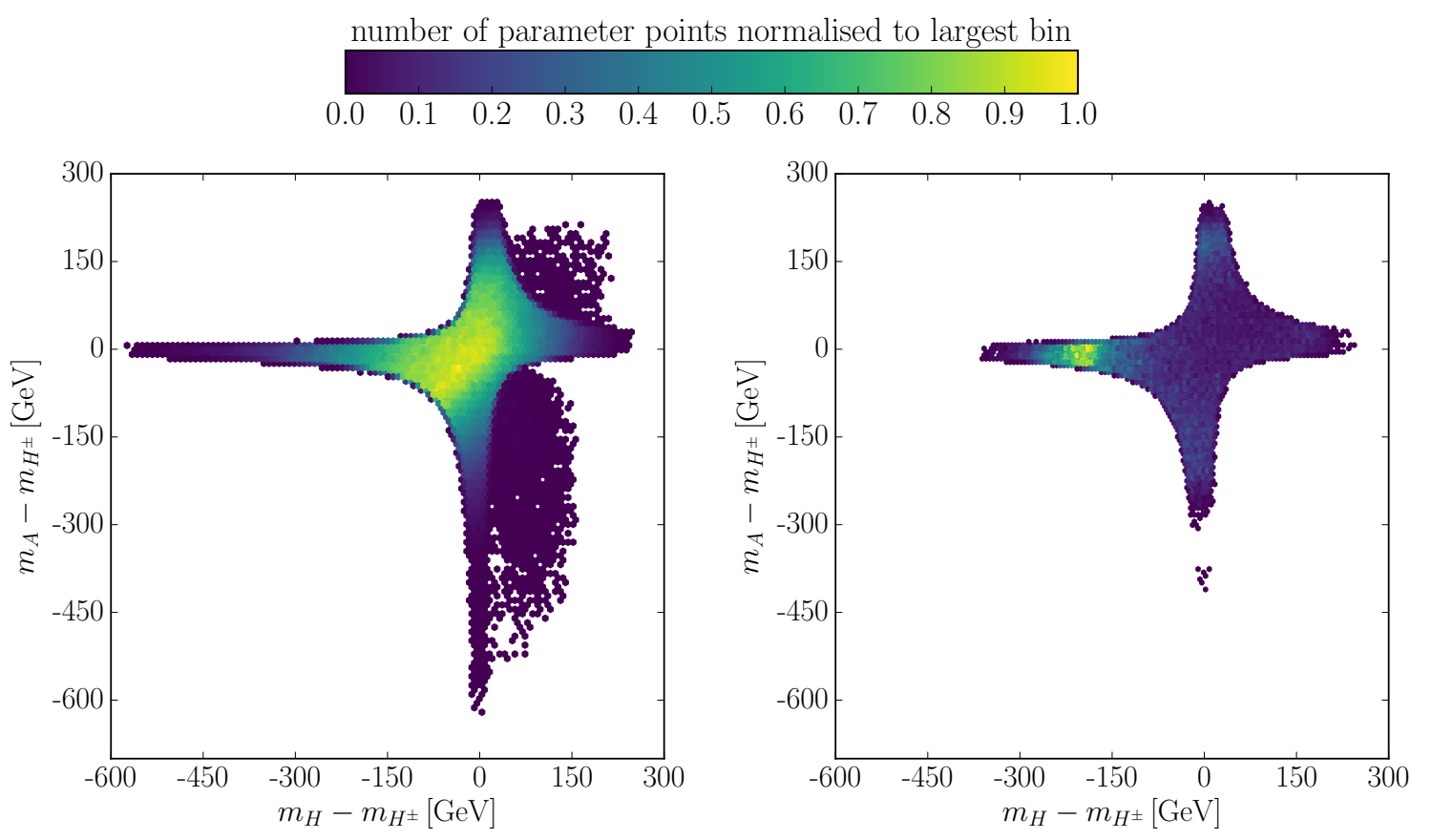

Figure 6. Type II, $h \equiv h_{125}$ : the mass difference $m_{A}-m_{H^{ \pm}}$versus $m_{H}-m_{H^{ \pm}}$. The colour code shows the relative frequency of left: all points passing the constraints; right: all points with additionally $\xi_{c} \geq 1$ ('Arnold-Espinosa' method).

cf. figure 6 (left). ${ }^{16}$ A strong first order PT, however, prefers scenarios with $m_{A} \approx m_{H^{ \pm}}$and with a large positive mass gap between $m_{H^{ \pm}}$and $m_{H}$ and hence also $m_{A}-m_{H} \gtrsim 180 \mathrm{GeV}$, cf. figure 6 (right). Scenarios where $m_{H} \approx m_{H^{ \pm}}$and $\left|m_{A}-m_{H^{ \pm}}\left(\approx m_{H}\right)\right|>0$ and also those where $m_{A} \approx m_{H^{ \pm}}$and $m_{H}-m_{H^{ \pm}}>0$ are rarer, as they would require a much heavier $H$, given that $m_{H^{ \pm}} \geq 480 \mathrm{GeV}$ in type II models. A heavy $H$ with non-vanishing VEV tends to reduce the strength of the phase transition. For the same reason scenarios where all non-SM-like Higgs bosons have similar masses are not very probable either. While again $A \rightarrow Z H$ is a typical decay that is possibly realised for strong first order PTs, the non-discovery of such a decay does not exclude $\xi_{c} \geq 1$ as other scenarios can be realised as well. We find that scenarios with $m_{A} \gtrsim 460 \mathrm{GeV}$ are preferred and namely those scenarios that are located in the alignment limit with $\tan \beta \approx 1$. This is, however, not due to the first order PT but already found by only imposing the theoretical and experimental constraints.

In the type II $2 \mathrm{HDM}$ there are parameter regions compatible with the experimental constraints where the coupling of the $h_{125}$ to the massive gauge bosons is of opposite sign with respect to the coupling to down-type fermions. This wrong-sign regime [81, $112,113]$ has interesting phenomenological implications like the non-decoupling of heavy particles $[77,112]$. Future precision measurements of the signal rates will allow to constrain or exclude this parameter region $[81,112,114]$. The question arises to which extent the

\footnotetext{
${ }^{16}$ The dark blue points with non-zero mass gaps are points in the wrong-sign regime (see below). Due to the different coupling structure in the wrong-sign regime the constraints from the $\rho$ parameter have a different shape when projected into the plane of the plot.
} 


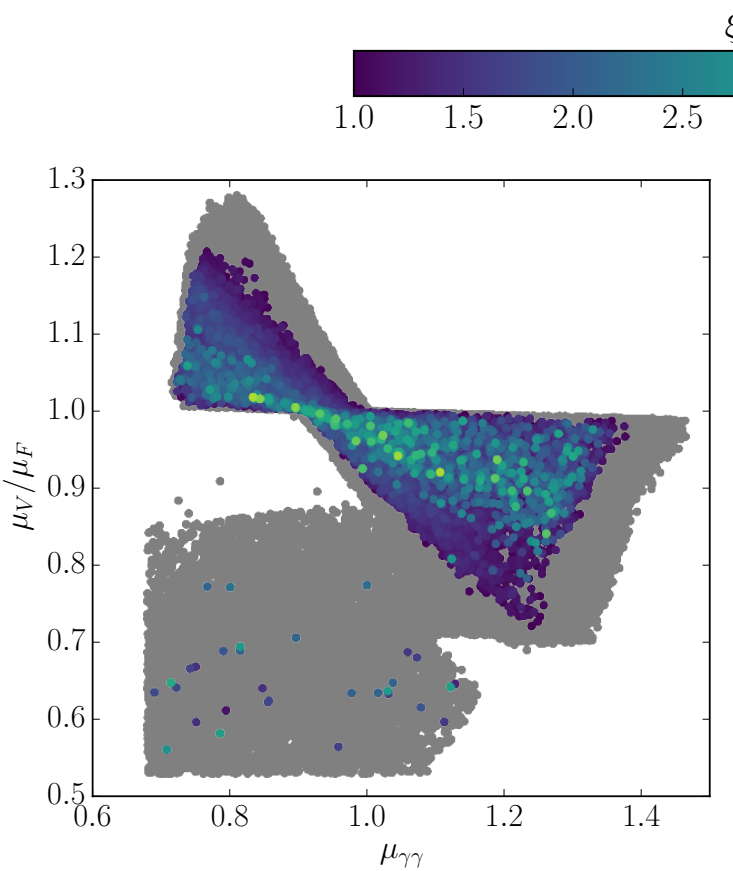

$\xi_{c}$

$\begin{array}{llll}1 & & & \\ 3.0 & 3.5 & 4.0 & 4.5\end{array}$

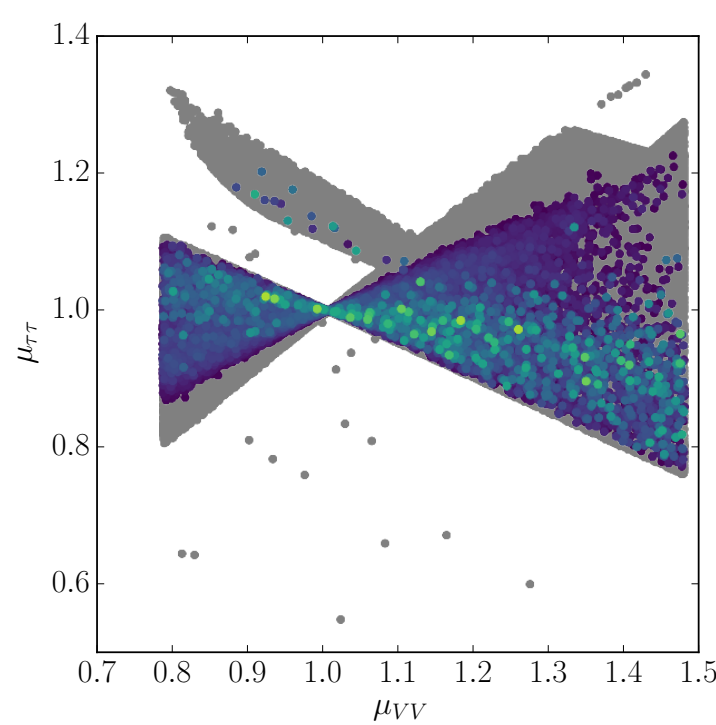

Figure 7. Type II, $h \equiv h_{125}: \mu_{V} / \mu_{F}$ versus $\mu_{\gamma \gamma}$ (left) and $\mu_{\tau \tau}$ versus $\mu_{V V}$ (right); grey: all points passing the applied constraints, colour: all points with additionally $\xi_{c} \geq 1$ ('Arnold-Espinosa' method). The colour code indicates the value of $\xi_{c}$.

requirement of a strong $\mathrm{PT}$ is able to restrict the wrong-sign regime. Figure 7 (left) displays $\mu_{V} / \mu_{F}$ versus $\mu_{\gamma \gamma}$. Among the grey points, which show the scenarios passing all constraints, the outliers in the left bottom corner of the plot correspond to the wrong-sign regime. The coloured points fulfill $\xi_{c} \geq 1$ and show that a strong PT strongly disfavours the wrong-sign regime. This can also be observed in figure 7 (right) where the distribution of $\mu_{\tau \tau}$ versus $\mu_{V V}$ is displayed. The wrong-sign regime scenarios are given by the outliers in the upper left corner of the plot. This behaviour can be understood by the fact that the VEV $\langle H\rangle$ of the heavy CP-even Higgs normalised to the SM VEV for $h \equiv h_{125}$ is given by

$$
\frac{\langle H\rangle^{2}}{v^{2}}=\cos ^{2}(\beta-\alpha) .
$$

In the wrong-sign regime non-zero values of $\cos (\beta-\alpha)$ are still compatible with the data. This means that $H$ can take a significant fraction of the VEV and drive the PT. If $H$ is not light enough, the $\mathrm{PT}$ is reduced to values $\xi_{c}<1$. We also observe that the maximum value of $\mu_{\gamma \gamma}$ is reduced from about 1.46 to about 1.38 .

\subsection{Type I: parameter sets with $H \equiv h_{125}$}

We now investigate scenarios where the heavier of the two CP-even Higgs bosons is the SM-like Higgs boson, i.e. $H \equiv h_{125}$. Figure 8 displays in the $m_{A}$ versus $m_{h}$ plane in grey all points passing the constraints and in colour all parameter points also compatible with $\xi_{c} \geq 1$. First, we observe that independent of the strength of the PT, there are only few 


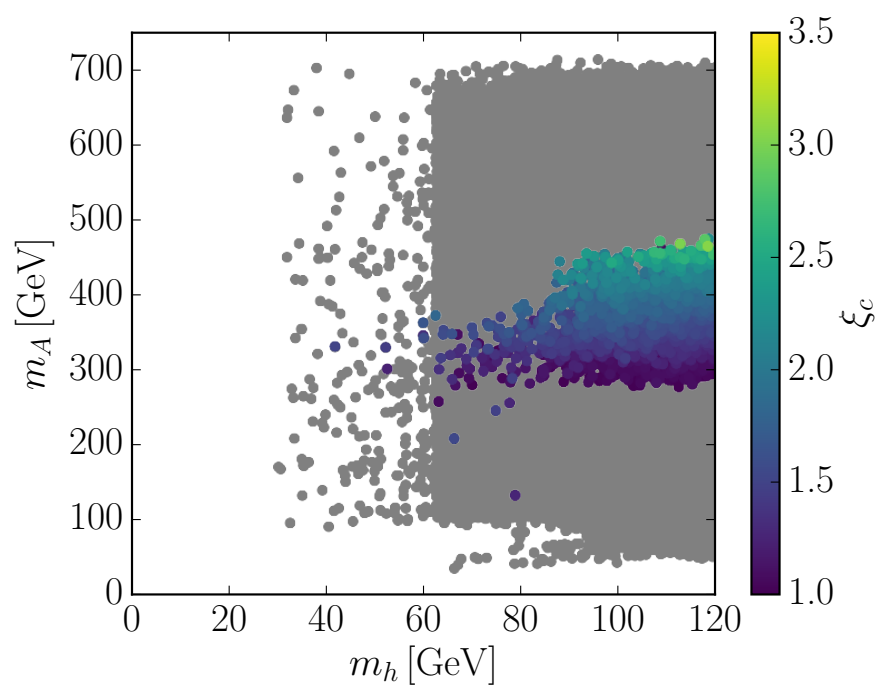

Figure 8. Type I, $H \equiv h_{125}$ : results in the $m_{A}$ versus $m_{h}$-plane, showing in grey the parameter points passing all applied constraints. Points highlighted in color have a PT of strong first order, where the value of $\xi_{c}$ is indicated by the color code ('Arnold-Espinosa' method).

scenarios with $m_{h} \lesssim 65 \mathrm{GeV}$. This is due to the fact that the decay $H \equiv h_{125} \rightarrow h h$ can change the total width of $h_{125}$ such that its branching ratios into SM final states lead to signal rates not compatible with the LHC data any more. In this mass region there are hardly any points with $\xi_{c} \geq 1$. The requirement of $\xi_{c} \geq 1$ also restricts the mass of the pseudocscalar to the region $280 \mathrm{GeV} \lesssim m_{A} \lesssim 480 \mathrm{GeV}$, with the exception of a few outliers. The strongest PTs are reached for larger $m_{A}$, close to $480 \mathrm{GeV}$. Figure 9 displays the distribution of the masses for $A$ and $H^{ \pm}$after applying all constraints (left) and when in addition $\xi_{c} \geq 1$ is demanded (right). With the exception of a few outliers, the strong PT restricts the mass region of the charged Higgs boson to $300 \mathrm{GeV} \lesssim m_{H^{ \pm}} \lesssim 480 \mathrm{GeV}$. As we demand the heavier of the two CP-even Higgs bosons to be light, the mass scale $m_{12}^{2}$, which determines its mass, cannot be too large. For the PT to be strong we need large quartic couplings. Since $\lambda_{2}$, which enters $m_{H}$, must not be large, we are left with $\lambda_{4}$ and $\lambda_{5}$ driving the PT, as can be inferred from the rather large mass values for $A$ and $H^{ \pm}$, namely the mass gap between $m_{H}$ and $m_{A, H^{ \pm}}$. When, on the other hand, the masses of the heavy Higgs bosons $A$ and $H^{ \pm}$become larger by increasing the involved quartic couplings the interplay of Higgs self-couplings and masses reduces $\xi_{c}$ again. We conclude that a strong $\mathrm{PT}$ in the $2 \mathrm{HDM}$ type I with two light CP-even Higgs boson excludes heavy Higgs bosons above about $500 \mathrm{GeV}$ and enforces a mass gap between $m_{A} \approx m_{H^{ \pm}}$and $m_{H}$. The decay $A \rightarrow H Z$, however, is suppressed because of $\sin (\beta-\alpha) \sim 0$ for $H \equiv h_{125}$. The decay $A \rightarrow h Z$ on the other hand, is allowed. For pseudoscalar masses above the top pair threshold, it competes, however, with the decay $A \rightarrow t \bar{t}$. 

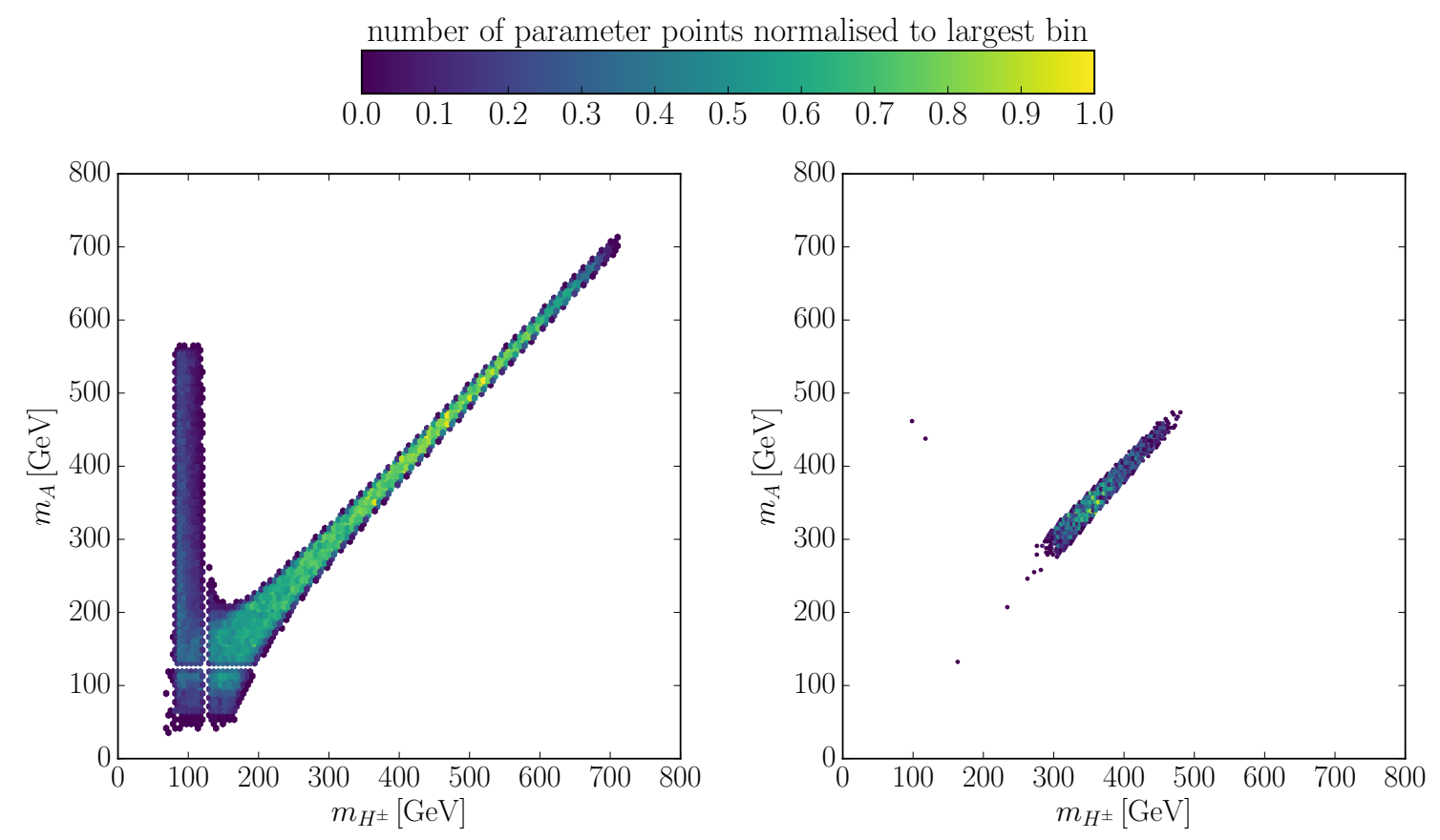

Figure 9. Type I, $H \equiv h_{125}$ : the masses $m_{A}$ versus $m_{H^{ \pm}}$. The colour code shows the relative frequency of left: all points passing the constraints; right: all points with additionally $\xi_{c} \geq 1$ ('Arnold-Espinosa' method).

The implications of a strong PT for the Higgs data are shown in figure 10. There are practically no points any more with values beyond 0.9 for the photonic rate, although rates of up to about 1.46 are still compatible with the Higgs data. Also the decays into $\tau$ final states cannot exceed 1.11 in case of $\xi_{c} \geq 1$.

\subsection{Type II: parameter sets with $H \equiv h_{125}$}

In the $2 \mathrm{HDM}$ type II with $H \equiv h_{125}$ the implications of a strong PT on the mass pattern are very pronounced, as can be inferred from figure 11. The requirement of $\xi_{c} \geq 1$ excludes a large portion of the parameter space, which is still compatible with the applied constraints. Scenarios with $m_{A} \approx m_{H^{ \pm}} \gtrsim 480 \mathrm{GeV}$ are forbidden if $\xi_{c} \geq 1$. Furthermore, very light scalars with $m_{h} \lesssim 110 \mathrm{GeV}$ are not compatible with a strong PT. The tension between the requirement of light scalar masses and the wish to have a strong PT makes a strong link between baryogenesis and collider phenomenology.

Further implications for LHC phenomenology are shown in figure 12 where the signal rates are displayed before (grey) and after (coloured) imposing a strong PT. All scenarios with $\xi_{c} \geq 1$ are located in the correct-sign regime (given by the triangle areas in the plots), whereas the wrong-sign regime (given by the outliers) is completely excluded by a strong PT. For the Higgs measurements, this means that the observation of $\mu_{V} / \mu_{F}<1$ together with $\mu_{\gamma \gamma} \lesssim 0.9$ is excluded, as well as the observation of $\mu_{\tau \tau} \gtrsim 1.04$. Furthermore, the region where $\mu_{\tau \tau} \lesssim 0.9$ because of possible decays $H \rightarrow h h$, is excluded by the demand of a strong PT. 


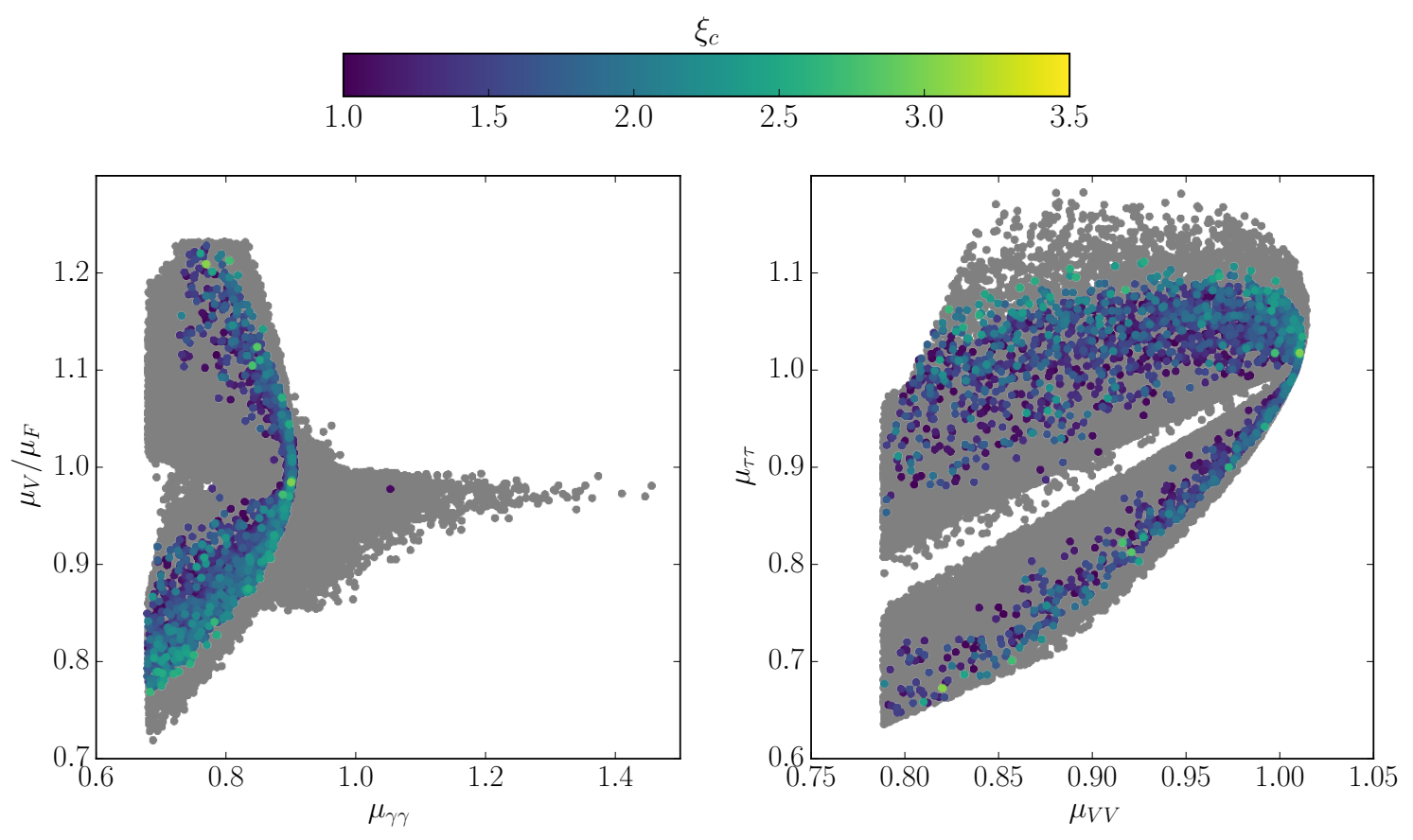

Figure 10. Type I, $H \equiv h_{125}: \mu_{V} / \mu_{F}$ versus $\mu_{\gamma \gamma}$ (left) and $\mu_{\tau \tau}$ versus $\mu_{V V}$ (right); grey: all points passing the applied constraints, colour: all points with additionally $\xi_{c} \geq 1$ ('Arnold-Espinosa' method). The colour code indicates the value of $\xi_{c}$.

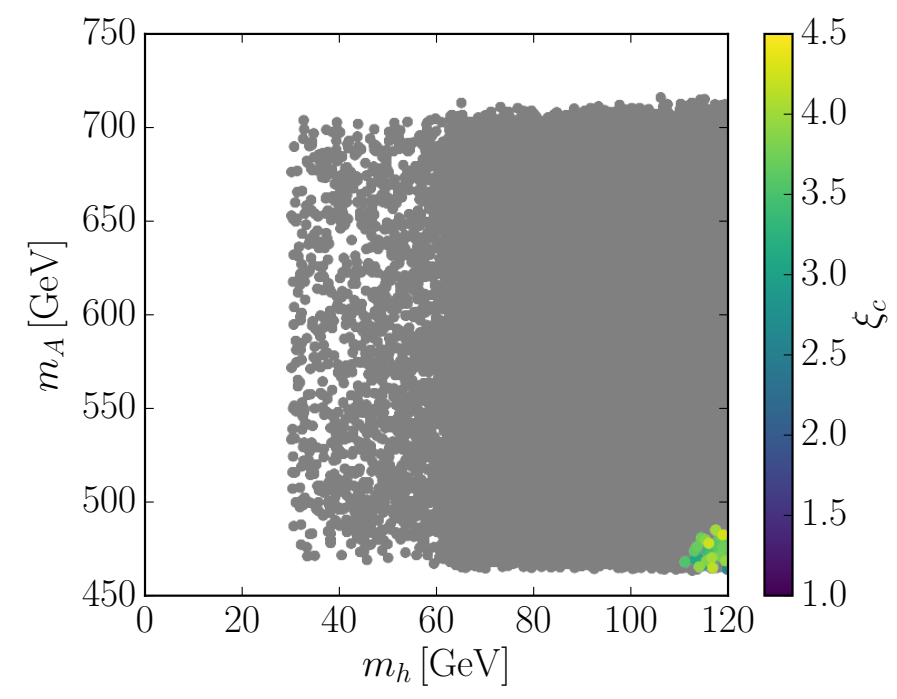

Figure 11. Type II, $H \equiv h_{125}$ : results in the $m_{A}$ versus $m_{H}$-plane, showing in grey the parameter points passing all applied constraints. Points highlighted in color have a PT of strong first order, where the value of $\xi_{c}$ is indicated by the color code ('Arnold-Espinosa' method). 
$\xi_{c}$

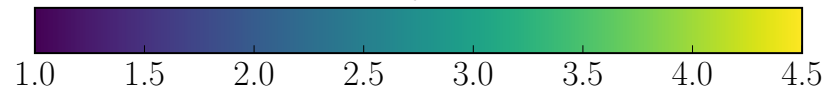
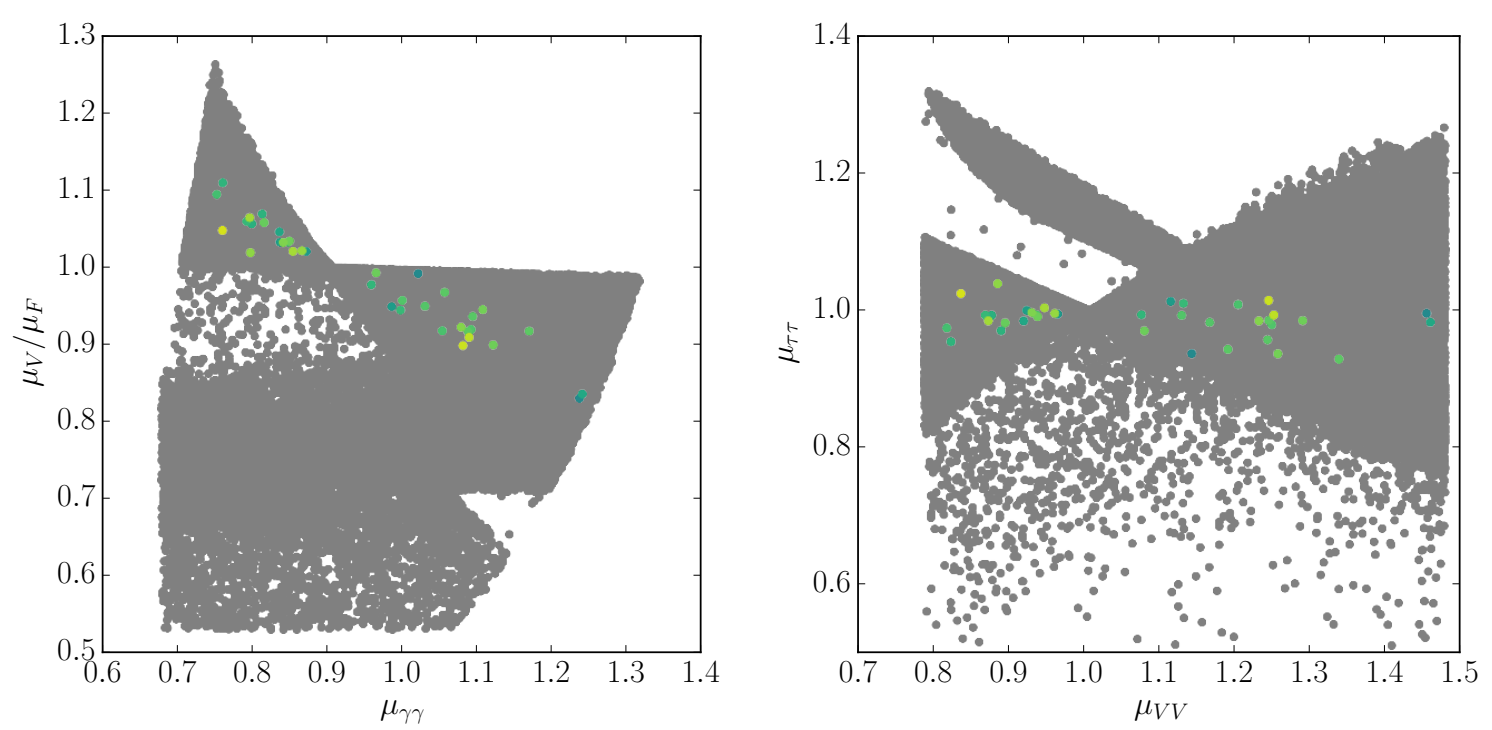

Figure 12. Type II, $H \equiv h_{125}: \mu_{V} / \mu_{F}$ versus $\mu_{\gamma \gamma}$ (left) and $\mu_{\tau \tau}$ versus $\mu_{V V}$ (right); grey: all points passing the applied constraints, colour: all points with additionally $\xi_{c} \geq 1$ ('Arnold-Espinosa' method). The colour code indicates the value of $\xi_{c}$.

\section{Conclusions}

In this paper we investigated the strength of the EW phase transition in the framework of the CP-conserving 2HDM. For this purpose we computed the loop-corrected effective potential at non-zero temperature including the resummation of the daisy graphs for the bosonic masses following the 'Arnold-Espinosa' method. We applied a renormalisation scheme that preserves the position of the minimum and where both the loop-corrected masses of the Higgs bosons and the mixing angles are renormalised to their tree-level values. This is in contrast to earlier works which focus solely on the Higgs boson masses. Our renormalisation allows us to efficiently scan the whole $2 \mathrm{HDM}$ parameter space and test the compatibility of the model with the theoretical and experimental constraints. This is possible since our renormalisation fixes not only the Higgs mass values but, through the mixing angles, also the Higgs couplings to their tree-level values.

We performed an extensive scan in the parameter space of the 2HDM and retained only those points that are compatible with the state-of-art theoretical and experimental constraints. For these parameter points we determined the value of $\xi_{c}$. Subsequently, we performed a comprehensive and systematic analysis in four $2 \mathrm{HDM}$ configurations: for the 2HDM type I and II, with either $h$ or $H$ identified with the SM-like Higgs boson, we investigated the implications of the requirement of a strong PT, i.e. $\xi_{c} \geq 1$, for LHC phenomenology. Our results can be summarised as follows: both the 2HDM type I and type II, with either of the CP-even Higgs bosons being the SM-like Higgs boson, are found 
to be compatible with the theoretical and experimental constraints on the model and a strong first order PT. The strong PT, however, strongly constrains the enhanced rates into photonic final states for the 2HDM type I, and to some extent also for type II. Furthermore, in the $2 \mathrm{HDM}$ type II with $h=h_{125}$ the wrong-sign regime is strongly restricted by the requirement of $\xi_{c} \geq 1$. In case $H=h_{125}$ the wrong-sign regime is even excluded for a strong PT. In more detail, our results for the four different realisations of the 2HDM are:

- For the $2 \mathrm{HDM}$ type I with $h \equiv h_{125}$, we confirm earlier results which find that a large mass splitting between the heavy scalars is favourable for a strong PT. The preferred scenarios are the ones with $m_{A} \approx m_{H^{ \pm}} \approx 400-500 \mathrm{GeV}$ and $m_{A}-m_{H} \gtrsim 200 \mathrm{GeV}$. However, we also find that scenarios with different hierarchies among the heavy Higgs bosons (but at least one of $H$ and $A$ nearly mass degenerate with $H^{ \pm}$) or with degenerate heavy Higgs bosons $H, A$ and $H^{ \pm}$are allowed, though much less frequent. The maximally allowed photonic rate is reduced from 1.5 to 1.1 in case of $\xi_{c} \geq 1$.

- We find in the 2HDM type II with $h=h_{125}$ that scenarios with $130 \mathrm{GeV} \lesssim m_{A} \lesssim 340 \mathrm{GeV}$, which are already strongly constrained by LHC searches in $A \rightarrow Z h_{125}$, are completely excluded by the requirement of a strong PT. This requirement also restricts the wrong-sign regime considerably. The maximum value of $\mu_{\gamma \gamma}$ is reduced from about 1.46 to about 1.38 .

- In the $2 \mathrm{HDM}$ type I with two light CP-even Higgs bosons, namely $H \equiv h_{125}$, the heavy Higgs masses cannot exceed $480 \mathrm{GeV}$, although experimentally still allowed, if $\xi_{c} \geq 1$. Furthermore, this enforces a mass gap of about $155 \mathrm{GeV}$ between the heavier and the lighter Higgs bosons. A strong PT is found to exclude almost completely scenarios with $m_{h} \lesssim 65 \mathrm{GeV}$. It strongly reduces $\mu_{\gamma \gamma}$ from 1.46 down to 0.9 (with very few exceptions) and limits the $\tau$ final state rates to values below 1.11.

- In the 2HDM type II with $H \equiv h_{125}$ the tension between a light CP-even Higgs mass spectrum and a strong PT excludes large portions of the parameter space. The observation of heavy Higgs bosons with masses above $480 \mathrm{GeV}$ or of a light Higgs boson with a mass below $110 \mathrm{GeV}$ is excluded by the requirement of a strong PT. Furthermore, simultaneously reduced values of $\mu_{V} / \mu_{F}<1$ and $\mu_{\gamma \gamma} \lesssim 0.9$ are not compatible with $\xi_{c} \geq 1$, nor values of $\mu_{\tau \tau} \gtrsim 1.04$. The reason is that the wrong-sign regime is excluded by a strong PT. The requirement of a strong PT also excludes parameter regions with reduced $\mu_{\tau \tau} \lesssim 0.9$, resulting from Higgs-to-Higgs decays $H \rightarrow h h$, which contribute to the total width of the SM-like Higgs boson.

Our results show that there is a strong interplay between the requirement of successful baryogenesis and LHC phenomenology. The realisation of a strong EW phase transition leads to testable consequences for collider phenomenology. The systematic investigations performed in this work serve as basis for further analyses of the LHC phenomenology of 2HDM models featuring a strong EW phase transition. 


\section{Acknowledgments}

MM wants to thank Prof. Dr. Werner Bernreuther for suggesting the study of the 2HDM phase transition in her diploma thesis. This triggered a long time ago the realisation of this paper. We are particularly grateful to Marco Sampaio for many enlightening discussions. We also want to thank David Lopez-Val and Florian Staub for very helpful discussions. We are indebted to Rui Santos for comments on our draft. We furthermore thank José Eliel Camargo-Molina, Pedro Ferreira, Luminita Mihaila, Ben O'Leary, Michael Spira and Hanna Ziesche for useful comments. MK acknowledges financial support by the Graduiertenkolleg "GRK 1694: Elementarteilchenphysik bei höchster Energie und höchster Präzision". AW acknowledges financial support by the "Karlsruhe School of Elementary Particle and Astroparticle Physics: Science and Technology (KSETA)".

\section{A Masses with thermal corrections}

In the following we give the mass formulae for the SM particles in terms of the field configurations $\omega_{k}(k=1,2,3)$. The masses of the physical particles are obtained from these formulae when the $\omega_{k}$ take the values that minimise the loop-corrected effective potential $\tilde{V}$, eq. (3.1). At non-zero temperature these are the $\bar{\omega}_{k}$, which we obtain from the numerical determination of the global minimum of $\tilde{V}$ at fixed $T$. For $T=0$ they are given by the VEVs $v_{1}$ and $v_{2}$. We only need the tree-level relations for the masses. At non-zero temperature we furthermore include the Debye corrections to the masses of the scalars and the longitudinal gauge bosons stemming from the resummation of the daisy graphs. The mass formulae will be specified in the following.

\section{A.1 Fermion masses}

The fermion masses do not get a Debye correction, and therefore the mass squared of a fermion $f$ at temperature $T$ is given by

$$
m_{f}^{2}(T)=\frac{1}{2} y_{f}^{2}\left|\phi_{k}^{c, 0}\right|^{2}=m_{f}^{2}(T=0) \frac{\left|\phi_{k}^{c, 0}\right|^{2}}{v_{k}^{2}}
$$

where $y_{f}$ is the tree-level Yukawa coupling and $k=1,2$ denotes the classical constant field configuration doublet $\Phi_{k}^{c}$ to which the fermion couples. This depends on the type of the $2 \mathrm{HDM}$, cf. table 1. For the neutral components of the doublets we have

$$
\begin{aligned}
& \left|\phi_{1}^{c, 0}\right|^{2}=\omega_{1}^{2} \\
& \left|\phi_{2}^{c, 0}\right|^{2}=\omega_{2}^{2}+\omega_{3}^{2} .
\end{aligned}
$$

The fermion mass at $T=0$ is given by the tree-level VEV $v_{k}$ of the doublet $\Phi_{k}^{c}$ as

$$
m_{f}(T=0)=\frac{y_{f}}{\sqrt{2}} v_{k}
$$




\section{A.2 Gauge boson masses}

The longitudinal gauge bosons get a Debye correction to their mass matrix. The masses including the thermal corrections, denoted in section 2.2 by $\bar{m}$, in terms of the field configurations $\omega_{k}$ are given by

$$
\begin{aligned}
\bar{m}_{W}^{2} & =\frac{g^{2}}{4} \omega^{2}+2 g^{2} T^{2} \\
\bar{m}_{\gamma}^{2} & =\left(g^{2}+g^{\prime 2}\right)\left(T^{2}+\frac{\omega^{2}}{8}\right)-\frac{1}{8} \sqrt{\left(g^{2}-g^{\prime 2}\right)^{2}\left(64 T^{4}+16 T^{2} \omega^{2}\right)+\left(g^{2}+g^{\prime 2}\right)^{2} \omega^{4}} \\
\bar{m}_{Z}^{2} & =\left(g^{2}+g^{\prime 2}\right)\left(T^{2}+\frac{\omega^{2}}{8}\right)+\frac{1}{8} \sqrt{\left(g^{2}-g^{\prime 2}\right)^{2}\left(64 T^{4}+16 T^{2} \omega^{2}\right)+\left(g^{2}+g^{\prime 2}\right)^{2} \omega^{4}},
\end{aligned}
$$

where $g$ and $g^{\prime}$ denote the $\mathrm{SU}(2)_{L}$ and $\mathrm{U}(1)_{Y}$ gauge couplings, respectively, and

$$
\omega^{2}=\sum_{i=1,2,3} \omega_{i}^{2}
$$

Again, the physical masses are obtained for $\omega_{i} \equiv \bar{\omega}_{i}$, and at $T=0$ we recover the wellknown relations for the physical gauge boson masses $\left(v^{2}=v_{1}^{2}+v_{2}^{2}=\left.\sum_{i=1,2,3} \bar{\omega}_{i}^{2}\right|_{T=0}\right)$

$$
m_{W}^{2}=\frac{g^{2}}{4} v^{2}, \quad m_{Z}^{2}=\frac{g^{2}+g^{\prime 2}}{4} v^{2} \quad \text { and } \quad m_{\gamma}^{2}=0 .
$$

\section{A.3 Masses of the Higgs bosons}

The tree-level relations for the mass matrices of the Higgs bosons in the interaction basis in terms of the $\omega_{k}$ are obtained by differentiating the tree-level Higgs potential $V_{\text {tree }}$ eq. (2.2) twice with respect to the real interaction fields

$$
\phi_{i} \equiv\left\{\rho_{1}, \eta_{1}, \rho_{2}, \eta_{2}, \zeta_{1}, \psi_{1}, \zeta_{2}, \psi_{2}\right\}
$$

and replacing the fields with their classical constant field configurations

$$
\phi_{i}^{c} \equiv\left\{0,0,0,0, \omega_{1}, 0, \omega_{2}, \omega_{3}\right\}
$$

leading to the mass matrix

$$
(\mathcal{M})_{i j}=\left.\frac{1}{2} \frac{\partial^{2} V_{\text {tree }}}{\partial \phi_{i} \partial \phi_{j}}\right|_{\phi=\phi^{c}}
$$

The physical masses are given by the field values in the global minimum of the potential where $\omega_{k} \equiv \bar{\omega}_{k}$, which at $T=0$ reduces to $\left.\bar{\omega}_{1,2}\right|_{T=0}=v_{1,2}$ and $\left.\bar{\omega}_{3}\right|_{T=0}=0$. Because of charge conservation the mass matrix of eq. (A.12) decomposes into a $4 \times 4$ matrix $\mathcal{M}^{C}$ for the charged fields $\rho_{1}, \eta_{1}, \rho_{2}, \eta_{2}$ and a $4 \times 4$ matrix $\mathcal{M}^{N}$ for the neutral states $\zeta_{1}, \psi_{1}, \zeta_{2}, \psi_{2}$. In the $\mathrm{CP}$-conserving $2 \mathrm{HDM}$ the neutral $\mathrm{CP}$-even and $\mathrm{CP}$-odd fields do not mix so that the latter matrix further decomposes into two $2 \times 2$ matrices, one for the CP-even Higgs states $\zeta_{1,2}$ and one for the pseudoscalar states $\psi_{1,2}$. 
We introduce the following definitions

$$
\begin{aligned}
y_{t}^{2} & =\frac{2}{v_{2}^{2}} m_{t}^{2}(T=0) \\
y_{b}^{2} & = \begin{cases}\frac{2}{v_{2}^{2}} m_{b}^{2}(T=0) & \text { Type I \& Lepton Specific } \\
\frac{2}{v_{1}^{2}} m_{b}^{2}(T=0) & \text { Type II \& Flipped }\end{cases} \\
d_{1} & =\frac{1}{48}\left[12 \lambda_{1}+8 \lambda_{3}+4 \lambda_{4}+3\left(3 g^{2}+g^{\prime 2}\right)\right] \\
d_{2} & =\frac{1}{48}\left[12 \lambda_{2}+8 \lambda_{3}+4 \lambda_{4}+3\left(3 g^{2}+g^{\prime 2}\right)+12 y_{t}^{2}\right] \\
c_{1} & = \begin{cases}d_{1} & \text { Type I \& Lepton Specific } \\
d_{1}+\frac{1}{4} y_{b}^{2} & \text { Type II \& Flipped }\end{cases} \\
c_{2} & = \begin{cases}d_{2}+\frac{1}{4} y_{b}^{2} & \text { Type I \& Lepton Specific } \\
d_{2} & \text { Type II \& Flipped }\end{cases}
\end{aligned}
$$

where we take for the top and bottom quark masses at zero temperature, $m_{t, b}(T=0)$, the input values given in eqs. (4.12) and (4.13). The masses of the charged Higgs boson and the charged Goldstone boson including the thermal corrections are then given by

$$
\begin{aligned}
\bar{m}_{H^{ \pm}}^{2}= & \frac{1}{2}\left(\mathcal{M}_{11}^{C}+\mathcal{M}_{22}^{C}+\left(c_{1}+c_{2}\right) T^{2}\right) \\
& +\frac{1}{2} \sqrt{\left(\mathcal{M}_{11}^{C}-\mathcal{M}_{22}^{C}+\left(c_{1}-c_{2}\right) T^{2}\right)^{2}+4\left(\left(\mathcal{M}_{12}^{C}\right)^{2}+\left(\mathcal{M}_{13}^{C}\right)^{2}\right)} \\
\bar{m}_{G^{ \pm}}^{2}= & \frac{1}{2}\left(\mathcal{M}_{11}^{C}+\mathcal{M}_{22}^{C}+\left(c_{1}+c_{2}\right) T^{2}\right) \\
& -\frac{1}{2} \sqrt{\left(\mathcal{M}_{11}^{C}-\mathcal{M}_{22}^{C}+\left(c_{1}-c_{2}\right) T^{2}\right)^{2}+4\left(\left(\mathcal{M}_{12}^{C}\right)^{2}+\left(\mathcal{M}_{13}^{C}\right)^{2}\right)},
\end{aligned}
$$

with

$$
\begin{aligned}
& \mathcal{M}_{11}^{C}=m_{11}^{2}+\lambda_{1} \frac{\omega_{1}^{2}}{2}+\lambda_{3} \frac{\omega_{2}^{2}+\omega_{3}^{2}}{2} \\
& \mathcal{M}_{22}^{C}=m_{22}^{2}+\lambda_{2} \frac{\omega_{2}^{2}+\omega_{3}^{2}}{2}+\lambda_{3} \frac{\omega_{1}^{2}}{2} \\
& \mathcal{M}_{12}^{C}=\frac{\omega_{1} \omega_{2}}{2}\left(\lambda_{4}+\lambda_{5}\right)-m_{12}^{2} \\
& \mathcal{M}_{13}^{C}=\frac{\omega_{1} \omega_{3}}{2}\left(\lambda_{4}-\lambda_{5}\right) .
\end{aligned}
$$

The thermal masses of the neutral Higgs bosons are given as the eigenvalues of

$$
\overline{\mathcal{M}}^{N}=\left(\mathcal{M}^{N}\right)^{2}+T^{2} \operatorname{diag}\left(c_{1}, c_{1}, c_{2}, c_{2}\right)
$$


in the basis $\left(\zeta_{1}, \psi_{1}, \zeta_{2}, \psi_{2}\right)$ with

$$
\begin{aligned}
& \mathcal{M}_{11}^{N}=m_{11}^{2}+\frac{3}{2} \lambda_{1} \omega_{1}^{2}+\frac{\lambda_{3}+\lambda_{4}}{2}\left(\omega_{2}^{2}+\omega_{3}^{2}\right)+\frac{1}{2} \lambda_{5}\left(\omega_{2}^{2}-\omega_{3}^{2}\right) \\
& \mathcal{M}_{22}^{N}=m_{11}+\frac{\lambda_{1}}{2} \omega_{1}^{2}+\frac{\lambda_{3}+\lambda_{4}}{2}\left(\omega_{2}^{2}+\omega_{3}^{2}\right)-\frac{1}{2} \lambda_{5}\left(\omega_{2}^{2}-\omega_{3}^{2}\right) \\
& \mathcal{M}_{33}^{N}=m_{22}^{2}+\frac{1}{2} \lambda_{2}\left(3 \omega_{2}^{2}+\omega_{3}^{2}\right)+\frac{1}{2}\left(\lambda_{3}+\lambda_{4}+\lambda_{5}\right) \omega_{1}^{2} \\
& \mathcal{M}_{44}^{N}=m_{22}^{2}+\frac{\lambda_{2}}{2}\left(\omega_{2}^{2}+3 \omega_{3}^{2}\right)+\frac{1}{2}\left(\lambda_{3}+\lambda_{4}-\lambda_{5}\right) \omega_{1}^{2} \\
& \mathcal{M}_{12}^{N}=\lambda_{5} \omega_{2} \omega_{3} \\
& \mathcal{M}_{13}^{N}=-m_{12}^{2}+\left(\lambda_{3}+\lambda_{4}+\lambda_{5}\right) \omega_{1} \omega_{2} \\
& \mathcal{M}_{14}^{N}=\left(\lambda_{3}+\lambda_{4}-\lambda_{5}\right) \omega_{1} \omega_{3} \\
& \mathcal{M}_{23}^{N}=\lambda_{5} \omega_{1} \omega_{3} \\
& \mathcal{M}_{24}^{N}=-m_{12}^{2}+\lambda_{5} \omega_{1} \omega_{2} \\
& \mathcal{M}_{34}^{N}=\lambda_{2} \omega_{2} \omega_{3} .
\end{aligned}
$$

The physical masses at $T=0$ are recovered after replacing the $\omega_{k}$ with the VEVs at $T=0$. In particular, the Goldstone masses become zero in the Landau gauge, in which we are working.

Open Access. This article is distributed under the terms of the Creative Commons Attribution License (CC-BY 4.0), which permits any use, distribution and reproduction in any medium, provided the original author(s) and source are credited.

\section{References}

[1] ATLAS collaboration, Observation of a new particle in the search for the Standard Model Higgs boson with the ATLAS detector at the LHC, Phys. Lett. B 716 (2012) 1 [arXiv:1207.7214] [INSPIRE].

[2] ATLAS collaboration, Updated ATLAS results on the signal strength of the Higgs-like boson for decays into $W W$ and heavy fermion final states, ATLAS-CONF-2012-162.

[3] CMS collaboration, Observation of a new boson at a mass of $125 \mathrm{GeV}$ with the CMS experiment at the LHC, Phys. Lett. B 716 (2012) 30 [arXiv:1207.7235] [InSPIRE].

[4] CMS collaboration, Combination of standard model Higgs boson searches and measurements of the properties of the new boson with a mass near $125 \mathrm{GeV}$, CMS-PAS-HIG-12-045.

[5] ATLAS collaboration, Study of the spin and parity of the Higgs boson in diboson decays with the ATLAS detector, Eur. Phys. J. C 75 (2015) 476 [arXiv:1506.05669] [INSPIRE].

[6] CMS collaboration, Constraints on the spin-parity and anomalous HVV couplings of the Higgs boson in proton collisions at 7 and 8 TeV, Phys. Rev. D 92 (2015) 012004 [arXiv: 1411.3441] [INSPIRE].

[7] ATLAS collaboration, Measurements of the Higgs boson production and decay rates and coupling strengths using pp collision data at $\sqrt{s}=7$ and $8 \mathrm{TeV}$ in the ATLAS experiment, Eur. Phys. J. C 76 (2016) 6 [arXiv:1507.04548] [InSPIRE]. 
[8] CMS collaboration, Precise determination of the mass of the Higgs boson and tests of compatibility of its couplings with the standard model predictions using proton collisions at 7 and $8 \mathrm{TeV}$, Eur. Phys. J. C 75 (2015) 212 [arXiv:1412.8662] [INSPIRE].

[9] WMAP collaboration, C.L. Bennett et al., Nine-Year Wilkinson Microwave Anisotropy Probe (WMAP) Observations: Final Maps and Results, Astrophys. J. Suppl. 208 (2013) 20 [arXiv: 1212.5225] [INSPIRE].

[10] V.A. Kuzmin, V.A. Rubakov and M.E. Shaposhnikov, On the Anomalous Electroweak Baryon Number Nonconservation in the Early Universe, Phys. Lett. B 155 (1985) 36 [INSPIRE].

[11] A.G. Cohen, D.B. Kaplan and A.E. Nelson, Baryogenesis at the weak phase transition, Nucl. Phys. B 349 (1991) 727 [inSPIRE].

[12] A.G. Cohen, D.B. Kaplan and A.E. Nelson, Progress in electroweak baryogenesis, Ann. Rev. Nucl. Part. Sci. 43 (1993) 27 [hep-ph/9302210] [InSPIRE].

[13] M. Quirós, Field theory at finite temperature and phase transitions, Helv. Phys. Acta 67 (1994) 451 [INSPIRE].

[14] V.A. Rubakov and M.E. Shaposhnikov, Electroweak baryon number nonconservation in the early universe and in high-energy collisions, Usp. Fiz. Nauk 166 (1996) 493 [hep-ph/9603208] [INSPIRE].

[15] K. Funakubo, CP violation and baryogenesis at the electroweak phase transition, Prog. Theor. Phys. 96 (1996) 475 [hep-ph/9608358] [INSPIRE].

[16] M. Trodden, Electroweak baryogenesis, Rev. Mod. Phys. 71 (1999) 1463 [hep-ph/9803479] [INSPIRE].

[17] W. Bernreuther, CP violation and baryogenesis, Lect. Notes Phys. 591 (2002) 237 [hep-ph/0205279] [INSPIRE].

[18] D.E. Morrissey and M.J. Ramsey-Musolf, Electroweak baryogenesis, New J. Phys. 14 (2012) 125003 [arXiv: 1206.2942 ] [INSPIRE].

[19] A.D. Sakharov, Violation of CP Invariance, c Asymmetry and Baryon Asymmetry of the Universe, Pisma Zh. Eksp. Teor. Fiz. 5 (1967) 32 [INSPIRE].

[20] N.S. Manton, Topology in the Weinberg-Salam Theory, Phys. Rev. D 28 (1983) 2019 [INSPIRE].

[21] F.R. Klinkhamer and N.S. Manton, A Saddle Point Solution in the Weinberg-Salam Theory, Phys. Rev. D 30 (1984) 2212 [InSPIRE].

[22] T. Konstandin, Quantum Transport and Electroweak Baryogenesis, Phys. Usp. 56 (2013) 747 [arXiv: 1302.6713] [INSPIRE].

[23] K. Kajantie, K. Rummukainen and M.E. Shaposhnikov, A Lattice Monte Carlo study of the hot electroweak phase transition, Nucl. Phys. B 407 (1993) 356 [hep-ph/9305345] [INSPIRE].

[24] Z. Fodor, J. Hein, K. Jansen, A. Jaster and I. Montvay, Simulating the electroweak phase transition in the SU(2) Higgs model, Nucl. Phys. B 439 (1995) 147 [hep-lat/9409017] [INSPIRE].

[25] K. Kajantie, M. Laine, K. Rummukainen and M.E. Shaposhnikov, The electroweak phase transition: a nonperturbative analysis, Nucl. Phys. B 466 (1996) 189 [hep-lat/9510020] [INSPIRE]. 
[26] K. Jansen, Status of the finite temperature electroweak phase transition on the lattice, Nucl. Phys. Proc. Suppl. 47 (1996) 196 [hep-lat/9509018] [INSPIRE].

[27] K. Kajantie, M. Laine, K. Rummukainen and M.E. Shaposhnikov, Is there a hot electroweak phase transition at $m_{H}$ larger or equal to $m_{W}$ ?, Phys. Rev. Lett. 77 (1996) 2887 [hep-ph/9605288] [INSPIRE].

[28] F. Csikor, Z. Fodor and J. Heitger, Endpoint of the hot electroweak phase transition, Phys. Rev. Lett. 82 (1999) 21 [hep-ph/9809291] [INSPIRE].

[29] J.M. Cline, Baryogenesis, hep-ph/0609145 [INSPIRE].

[30] T.D. Lee, A Theory of Spontaneous T Violation, Phys. Rev. D 8 (1973) 1226 [INSPIRE].

[31] G.C. Branco, P.M. Ferreira, L. Lavoura, M.N. Rebelo, M. Sher and J.P. Silva, Theory and phenomenology of two-Higgs-doublet models, Phys. Rept. 516 (2012) 1 [arXiv:1106.0034] [INSPIRE].

[32] A.I. Bochkarev, S.V. Kuzmin and M.E. Shaposhnikov, Electroweak baryogenesis and the Higgs boson mass problem, Phys. Lett. B 244 (1990) 275 [INSPIRE].

[33] L.D. McLerran, M.E. Shaposhnikov, N. Turok and M.B. Voloshin, Why the baryon asymmetry of the universe is approximately $10^{-10}$, Phys. Lett. B 256 (1991) 451 [InSPIRE].

[34] A.I. Bochkarev, S.V. Kuzmin and M.E. Shaposhnikov, On the Model Dependence of the Cosmological Upper Bound on the Higgs Boson and Top Quark Masses, Phys. Rev. D 43 (1991) 369 [INSPIRE].

[35] N. Turok and J. Zadrozny, Electroweak baryogenesis in the two doublet model, Nucl. Phys. B 358 (1991) 471 [INSPIRE].

[36] A.G. Cohen, D.B. Kaplan and A.E. Nelson, Spontaneous baryogenesis at the weak phase transition, Phys. Lett. B 263 (1991) 86 [InSPIRE].

[37] N. Turok and J. Zadrozny, Phase transitions in the two doublet model, Nucl. Phys. B 369 (1992) 729 [INSPIRE].

[38] A.E. Nelson, D.B. Kaplan and A.G. Cohen, Why there is something rather than nothing: Matter from weak interactions, Nucl. Phys. B 373 (1992) 453 [INSPIRE].

[39] K. Funakubo, A. Kakuto and K. Takenaga, The effective potential of electroweak theory with two massless Higgs doublets at finite temperature, Prog. Theor. Phys. 91 (1994) 341 [hep-ph/9310267] [INSPIRE].

[40] A.T. Davies, C.D. froggatt, G. Jenkins and R.G. Moorhouse, Baryogenesis constraints on two Higgs doublet models, Phys. Lett. B 336 (1994) 464 [inSPIRE].

[41] K. Funakubo, A. Kakuto, S. Otsuki, K. Takenaga and F. Toyoda, CP violating profile of the electroweak bubble wall, Prog. Theor. Phys. 94 (1995) 845 [hep-ph/9507452] [INSPIRE].

[42] K. Funakubo, A. Kakuto, S. Otsuki and F. Toyoda, Explicit CP breaking and electroweak baryogenesis, Prog. Theor. Phys. 96 (1996) 771 [hep-ph/9606282] [InSPIRE].

[43] J.M. Cline, K. Kainulainen and A.P. Vischer, Dynamics of two Higgs doublet CP-violation and baryogenesis at the electroweak phase transition, Phys. Rev. D 54 (1996) 2451 [hep-ph/9506284] [INSPIRE].

[44] K. Fuyuto and E. Senaha, Sphaleron and critical bubble in the scale invariant two Higgs doublet model, Phys. Lett. B 747 (2015) 152 [INSPIRE].

[45] C.-W. Chiang, K. Fuyuto and E. Senaha, Electroweak Baryogenesis with Lepton Flavor Violation, Phys. Lett. B 762 (2016) 315 [arXiv:1607.07316] [INSPIRE]. 
[46] G.C. Dorsch, S.J. Huber and J.M. No, A strong electroweak phase transition in the 2HDM after LHC8, JHEP 10 (2013) 029 [arXiv: 1305.6610] [INSPIRE].

[47] G.C. Dorsch, S.J. Huber, K. Mimasu and J.M. No, Echoes of the Electroweak Phase Transition: Discovering a second Higgs doublet through $A_{0} \rightarrow Z H_{0}$, Phys. Rev. Lett. 113 (2014) 211802 [arXiv:1405.5537] [INSPIRE].

[48] J.M. Cline and P.-A. Lemieux, Electroweak phase transition in two Higgs doublet models, Phys. Rev. D 55 (1997) 3873 [hep-ph/9609240] [INSPIRE].

[49] L. Fromme, S.J. Huber and M. Seniuch, Baryogenesis in the two-Higgs doublet model, JHEP 11 (2006) 038 [hep-ph/0605242] [INSPIRE].

[50] J.M. Cline, K. Kainulainen and M. Trott, Electroweak Baryogenesis in Two Higgs Doublet Models and B meson anomalies, JHEP 11 (2011) 089 [arXiv:1107.3559] [INSPIRE].

[51] G.C. Dorsch, S.J. Huber, T. Konstandin and J.M. No, A Second Higgs Doublet in the Early Universe: Baryogenesis and Gravitational Waves, arXiv:1611.05874 [INSPIRE].

[52] A. Haarr, A. Kvellestad and T.C. Petersen, Disfavouring Electroweak Baryogenesis and a hidden Higgs in a CP-violating Two-Higgs-Doublet Model, arXiv:1611.05757 [INSPIRE].

[53] S.R. Coleman and E.J. Weinberg, Radiative Corrections as the Origin of Spontaneous Symmetry Breaking, Phys. Rev. D 7 (1973) 1888 [inSPIRE].

[54] L. Dolan and R. Jackiw, Symmetry Behavior at Finite Temperature, Phys. Rev. D 9 (1974) 3320 [INSPIRE].

[55] M. Quirós, Finite temperature field theory and phase transitions, hep-ph/9901312 [INSPIRE].

[56] M.E. Carrington, The effective potential at finite temperature in the Standard Model, Phys. Rev. D 45 (1992) 2933 [INSPIRE].

[57] P.B. Arnold and O. Espinosa, The Effective potential and first order phase transitions: Beyond leading-order, Phys. Rev. D 47 (1993) 3546 [Erratum ibid. D 50 (1994) 6662] [hep-ph/9212235] [INSPIRE].

[58] R.R. Parwani, Resummation in a hot scalar field theory, Phys. Rev. D 45 (1992) 4695 [Erratum ibid. D 48 (1993) 5965] [hep-ph/9204216] [INSPIRE].

[59] H.H. Patel and M.J. Ramsey-Musolf, Baryon Washout, Electroweak Phase Transition and Perturbation Theory, JHEP 07 (2011) 029 [arXiv:1101.4665] [INSPIRE].

[60] C. Wainwright, S. Profumo and M.J. Ramsey-Musolf, Gravity Waves from a Cosmological Phase Transition: Gauge Artifacts and Daisy Resummations, Phys. Rev. D 84 (2011) 023521 [arXiv: 1104.5487] [INSPIRE].

[61] M. Garny and T. Konstandin, On the gauge dependence of vacuum transitions at finite temperature, JHEP 07 (2012) 189 [arXiv:1205.3392] [INSPIRE].

[62] G.D. Moore, Measuring the broken phase sphaleron rate nonperturbatively, Phys. Rev. D 59 (1999) 014503 [hep-ph/9805264] [INSPIRE].

[63] D. Land and E.D. Carlson, Two stage phase transition in two Higgs models, Phys. Lett. B 292 (1992) 107 [hep-ph/9208227] [INSPIRE].

[64] A. Hammerschmitt, J. Kripfganz and M.G. Schmidt, Baryon asymmetry from a two stage electroweak phase transition?, Z. Phys. C 64 (1994) 105 [hep-ph/9404272] [INSPIRE]. 
[65] P.M. Ferreira, R. Santos and A. Barroso, Stability of the tree-level vacuum in two Higgs doublet models against charge or CP spontaneous violation, Phys. Lett. B 603 (2004) 219 [Erratum ibid. B 629 (2005) 114] [hep-ph/0406231] [INSPIRE].

[66] A. Barroso, P.M. Ferreira and R. Santos, Neutral minima in two-Higgs doublet models, Phys. Lett. B 652 (2007) 181 [hep-ph/0702098] [INSPIRE].

[67] I.P. Ivanov, Minkowski space structure of the Higgs potential in 2HDM. II. Minima, symmetries and topology, Phys. Rev. D 77 (2008) 015017 [arXiv:0710.3490] [INSPIRE].

[68] P.M. Ferreira and B. Swiezewska, One-loop contributions to neutral minima in the inert doublet model, JHEP 04 (2016) 099 [arXiv: 1511.02879] [INSPIRE].

[69] D. Fontes, J.C. Romão, R. Santos and J.P. Silva, Large pseudoscalar Yukawa couplings in the complex 2HDM, JHEP 06 (2015) 060 [arXiv:1502.01720] [INSPIRE].

[70] M. Carena, G. Nardini, M. Quirós and C.E.M. Wagner, The Baryogenesis Window in the MSSM, Nucl. Phys. B 812 (2009) 243 [arXiv:0809.3760] [INSPIRE].

[71] E.J. Weinberg and A.-q. Wu, Understanding complex perturbative effective potentials, Phys. Rev. D 36 (1987) 2474 [INSPIRE].

[72] J.E. Camargo-Molina, A.P. Morais, R. Pasechnik, M.O.P. Sampaio and J. Wessén, All one-loop scalar vertices in the effective potential approach, JHEP 08 (2016) 073 [arXiv: 1606. 07069] [INSPIRE].

[73] S.P. Martin, Taming the Goldstone contributions to the effective potential, Phys. Rev. D 90 (2014) 016013 [arXiv: 1406.2355] [INSPIRE].

[74] J. Elias-Miro, J.R. Espinosa and T. Konstandin, Taming Infrared Divergences in the Effective Potential, JHEP 08 (2014) 034 [arXiv: 1406.2652] [InSPIRE].

[75] J.A. Casas, J.R. Espinosa, M. Quirós and A. Riotto, The Lightest Higgs boson mass in the minimal supersymmetric standard model, Nucl. Phys. B 436 (1995) 3 [Erratum ibid. B 439 (1995) 466] [hep-ph/9407389] [INSPIRE].

[76] M. Krause, R. Lorenz, M. Muhlleitner, R. Santos and H. Ziesche, Gauge-independent Renormalization of the 2-Higgs-Doublet Model, JHEP 09 (2016) 143 [arXiv:1605.04853] [INSPIRE].

[77] M. Krause, M. Muhlleitner, R. Santos and H. Ziesche, 2HDM Higgs-to-Higgs Decays at Next-to-Leading Order, arXiv:1609.04185 [INSPIRE].

[78] E. Benazera and N. Hansen, libcmaes, https://github.com/beniz/libcmaes.

[79] M. Galassi et al., GNU Scientific Library Reference Manual, 3rd edition, http://www.gnu.org/software/gsl/.

[80] R. Coimbra, M.O.P. Sampaio and R. Santos, ScannerS: Constraining the phase diagram of a complex scalar singlet at the LHC, Eur. Phys. J. C 73 (2013) 2428 [arXiv:1301.2599] [INSPIRE].

[81] P.M. Ferreira, R. Guedes, M.O.P. Sampaio and R. Santos, Wrong sign and symmetric limits and non-decoupling in 2HDMs, JHEP 12 (2014) 067 [arXiv:1409.6723] [INSPIRE].

[82] K.G. Klimenko, On Necessary and Sufficient Conditions for Some Higgs Potentials to Be Bounded From Below, Theor. Math. Phys. 62 (1985) 58 [InSPIRE].

[83] I.F. Ginzburg and I.P. Ivanov, Tree level unitarity constraints in the 2HDM with CP-violation, hep-ph/0312374 [INSPIRE]. 
[84] A. Barroso, P.M. Ferreira, I.P. Ivanov and R. Santos, Metastability bounds on the two Higgs doublet model, JHEP 06 (2013) 045 [arXiv: 1303.5098] [INSPIRE].

[85] M.E. Peskin and T. Takeuchi, Estimation of oblique electroweak corrections, Phys. Rev. D 46 (1992) 381 [INSPIRE].

[86] W. Grimus, L. Lavoura, O.M. Ogreid and P. Osland, The Oblique parameters in multi-Higgs-doublet models, Nucl. Phys. B 801 (2008) 81 [arXiv:0802.4353] [INSPIRE].

[87] W. Grimus, L. Lavoura, O.M. Ogreid and P. Osland, A Precision constraint on multi-Higgs-doublet models, J. Phys. G 35 (2008) 075001 [arXiv:0711.4022] [INSPIRE].

[88] GFITter Group collaboration, M. Baak et al., The global electroweak fit at NNLO and prospects for the LHC and ILC, Eur. Phys. J. C 74 (2014) 3046 [arXiv:1407.3792] [INSPIRE].

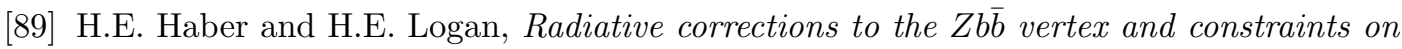
extended Higgs sectors, Phys. Rev. D 62 (2000) 015011 [hep-ph/9909335] [INSPIRE].

[90] O. Deschamps, S. Descotes-Genon, S. Monteil, V. Niess, S. T'Jampens and V. Tisserand, The Two Higgs Doublet of Type II facing flavour physics data, Phys. Rev. D 82 (2010) 073012 [arXiv: 0907.5135] [INSPIRE].

[91] F. Mahmoudi and O. Stål, Flavor constraints on the two-Higgs-doublet model with general Yukawa couplings, Phys. Rev. D 81 (2010) 035016 [arXiv:0907.1791] [INSPIRE].

[92] T. Hermann, M. Misiak and M. Steinhauser, $\bar{B} \rightarrow X_{s} \gamma$ in the Two Higgs Doublet Model up to Next-to-Next-to-Leading Order in QCD, JHEP 11 (2012) 036 [arXiv:1208.2788] [INSPIRE].

[93] M. Misiak et al., Updated NNLO QCD predictions for the weak radiative B-meson decays, Phys. Rev. Lett. 114 (2015) 221801 [arXiv:1503.01789] [INSPIRE].

[94] LEP, DELPHI, OPAL, ALEPH, L3 collaborations, G. Abbiendi et al., Search for Charged Higgs bosons: Combined Results Using LEP Data, Eur. Phys. J. C 73 (2013) 2463 [arXiv: 1301.6065] [INSPIRE].

[95] ATLAS collaboration, Search for charged Higgs bosons decaying via $H^{ \pm} \rightarrow \tau^{ \pm} \nu$ in fully hadronic final states using pp collision data at $\sqrt{s}=8 \mathrm{TeV}$ with the ATLAS detector, JHEP 03 (2015) 088 [arXiv: 1412.6663] [INSPIRE].

[96] CMS collaboration, Search for a charged Higgs boson in pp collisions at $\sqrt{s}=8 \mathrm{TeV}$, JHEP 11 (2015) 018 [arXiv: 1508.07774] [INSPIRE].

[97] ATLAS collaboration, Search for charged Higgs bosons in the $H^{ \pm} \rightarrow$ tb decay channel in pp collisions at $\sqrt{s}=8 \mathrm{TeV}$ using the ATLAS detector, JHEP 03 (2016) 127 [arXiv: 1512.03704] [INSPIRE].

[98] A. Djouadi, J. Kalinowski and M. Spira, HDECAY: A program for Higgs boson decays in the standard model and its supersymmetric extension, Comput. Phys. Commun. 108 (1998) 56 [hep-ph/9704448] [INSPIRE].

[99] J.M. Butterworth et al., The Tools And Monte Carlo Working Group Summary Report from the Les Houches 2009 Workshop on TeV Colliders, arXiv:1003.1643 [INSPIRE].

[100] R. Harlander, M. Mühlleitner, J. Rathsman, M. Spira and O. Stål, Interim recommendations for the evaluation of Higgs production cross sections and branching ratios at the LHC in the Two-Higgs-Doublet Model, arXiv:1312.5571 [INSPIRE]. 
[101] R.V. Harlander, S. Liebler and H. Mantler, SusHi: A program for the calculation of Higgs production in gluon fusion and bottom-quark annihilation in the Standard Model and the MSSM, Comput. Phys. Commun. 184 (2013) 1605 [arXiv:1212.3249] [InSPIRE].

[102] P. Bechtle, O. Brein, S. Heinemeyer, G. Weiglein and K.E. Williams, HiggsBounds: Confronting Arbitrary Higgs Sectors with Exclusion Bounds from LEP and the Tevatron, Comput. Phys. Commun. 181 (2010) 138 [arXiv:0811.4169] [InSPIRE].

[103] P. Bechtle, O. Brein, S. Heinemeyer, G. Weiglein and K.E. Williams, HiggsBounds 2.0.0: Confronting Neutral and Charged Higgs Sector Predictions with Exclusion Bounds from LEP and the Tevatron, Comput. Phys. Commun. 182 (2011) 2605 [arXiv:1102.1898] [InSPIRE].

[104] P. Bechtle et al., HiggsBounds - 4: Improved Tests of Extended Higgs Sectors against Exclusion Bounds from LEP, the Tevatron and the LHC, Eur. Phys. J. C 74 (2014) 2693 [arXiv: 1311.0055] [INSPIRE].

[105] ATLAS, CMS collaborations, Measurements of the Higgs boson production and decay rates and constraints on its couplings from a combined ATLAS and CMS analysis of the LHC pp collision data at $\sqrt{s}=7$ and $8 \mathrm{TeV}$, JHEP 08 (2016) 045 [arXiv: 1606.02266] [INSPIRE].

[106] ATLAS, CMS collaboration, Combined Measurement of the Higgs Boson Mass in pp Collisions at $\sqrt{s}=7$ and $8 \mathrm{TeV}$ with the ATLAS and CMS Experiments, Phys. Rev. Lett. 114 (2015) 191803 [arXiv: 1503.07589] [INSPIRE].

[107] Particle Data Group collaboration, K.A. Olive et al., Review of Particle Physics, Chin. Phys. C 38 (2014) 090001 [inSPIRE].

[108] A. Denner et al., Standard Model input parameters for Higgs physics, LHCHXSWG-INT-2015-006.

[109] LHC Higgs Cross Section Working Group, https://twiki.cern.ch/twiki/bin/view/LHCPhysics/LHCHXSWG.

[110] LHC Higgs Cross Section Working Group, S. Dittmaier et al., Handbook of LHC Higgs Cross sections: 1. Inclusive Observables, arXiv:1101.0593 [INSPIRE].

[111] J.F. Gunion and H.E. Haber, The CP conserving two Higgs doublet model: The Approach to the decoupling limit, Phys. Rev. D 67 (2003) 075019 [hep-ph/0207010] [INSPIRE].

[112] P.M. Ferreira, J.F. Gunion, H.E. Haber and R. Santos, Probing wrong-sign Yukawa couplings at the LHC and a future linear collider, Phys. Rev. D 89 (2014) 115003 [arXiv:1403.4736] [INSPIRE].

[113] D. Fontes, J.C. Romão and J.P. Silva, A reappraisal of the wrong-sign hbु coupling and the study of $h \rightarrow Z \gamma$, Phys. Rev. D 90 (2014) 015021 [arXiv:1406.6080] [INSPIRE].

[114] M. Muhlleitner, M.O.P. Sampaio, R. Santos and J. Wittbrodt, The N2HDM under Theoretical and Experimental Scrutiny, arXiv:1612.01309 [INSPIRE]. 